The University of Southern Mississippi

The Aquila Digital Community

Faculty Publications

$2-1-2019$

\title{
Diagonalization of 1-D Differential Operators With Piecewise Constant Coefficients Using the Uncertainty Principle
}

\author{
Sarah D. Long \\ University of Southern Mississippi \\ Somayyeh Sheikholeslami \\ Beaumont Health, s.sheikholeslami@usm.edu \\ James V. Lambers \\ University of Southern Mississippi, james.lambers@usm.edu \\ Carley Walker \\ University of Southern Mississippi
}

Follow this and additional works at: https://aquila.usm.edu/fac_pubs

Part of the Mathematics Commons

\section{Recommended Citation}

Long, S. D., Sheikholeslami, S., Lambers, J. V., Walker, C. (2019). Diagonalization of 1-D Differential Operators With Piecewise Constant Coefficients Using the Uncertainty Principle. Mathematics and Computers in Simulation, 156, 194-226.

Available at: https://aquila.usm.edu/fac_pubs/15542

This Article is brought to you for free and open access by The Aquila Digital Community. It has been accepted for inclusion in Faculty Publications by an authorized administrator of The Aquila Digital Community. For more information, please contact Joshua.Cromwell@usm.edu. 


\title{
Diagonalization of 1-D Differential Operators with Piecewise Constant Coefficients Using the Uncertainty Principle
}

\author{
Sarah D. Longa ${ }^{\mathrm{a}}$, Somayyeh Sheikholeslami ${ }^{\mathrm{b}}$, James V. Lambers ${ }^{\mathrm{a}}$, Carley \\ Walker $^{\mathrm{a}}$ \\ ${ }^{a}$ Department of Mathematics, The University of Southern Mississippi, 118 College Dr \\ \#5045, Hattiesburg, MS 39406 USA \\ ${ }^{b}$ Department of Radiation Oncology, Beaumont Health, Royal Oak, MI 48073 USA
}

\begin{abstract}
A highly accurate and efficient numerical method is presented for computing the solution of a 1-D time-dependent partial differential equation in which the spatial differential operator features a piecewise constant coefficient defined on $n$ pieces, in either self-adjoint and non-self-adjoint form, on a finite interval with periodic boundary conditions. The Uncertainty Principle is used to estimate the eigenvalues of the operator. Then, these estimates are used to construct a basis of eigenfunctions for use with a spectral method. The solution is presented as a truncated eigenfunction expansion, where each eigenfunction is a wave function that changes frequencies at the interfaces between different materials. Numerical experiments demonstrate the accuracy, efficiency and scalability of the method in comparison to other methods.
\end{abstract}

Keywords: heat equation, Uncertainty Principle, interface problem, spectral methods, eigenfunction expansion

\section{Introduction}

Let $\alpha(x)$ be a piecewise constant function on $[0,2 \pi)$, defined on $n$ pieces as follows:

$$
\alpha(x)=\left\{\begin{array}{cl}
\alpha_{1} & 0 \leq x<2 \pi \rho_{1} \\
\alpha_{2} & 2 \pi \rho_{1} \leq x<2 \pi \rho_{2} \\
\vdots & \vdots \\
\alpha_{n} & 2 \pi \rho_{n-1} \leq x<2 \pi
\end{array}\right.
$$


where $0<\rho_{1}<\rho_{2}<\cdots \rho_{n-1}<1$. This function therefore has jump discontinuities at $x_{i}=2 \pi \rho_{i}$ for $i=1,2, \ldots, n-1$. We consider the initial value problems

$$
u_{t}=\alpha^{2}(x) u_{x x}
$$

or

$$
u_{t}=\left(\alpha^{2}(x) u_{x}\right)_{x}
$$

on the domain $(0,2 \pi) \times(0, \infty)$, with an initial condition $u(x, 0)=f(x)$ and periodic boundary conditions. Throughout this paper, we use subscripts to denote partial derivatives. That is, $u_{t}=\partial u / \partial t$ and $u_{x x}=\partial^{2} u / \partial x^{2}$.

Equations (2) and (3) are special cases of the equation for heat transfer in a general medium,

$$
u_{t}=\frac{1}{c(x)^{2}}\left(a(x)^{2} u_{x}\right)
$$

where the coefficient $c(x)^{2}$ is the specific heat, and $a(x)^{2}$ is the diffusion coefficient that defines the heat flux $J(x)=a(x)^{2} u_{x}$. In equation (2), hereafter referred to as the non-self-adjoint case, we assume a constant diffusion coefficient, whereas in (3), hereafter referred to as the self-adjoint case, we assume a constant specific heat.

Simulation of phenomena such as diffusion of heat energy or wave propagation through multiple homogeneous media, represented by the piecewise constant coefficient $\alpha(x)$, is challenging for either analytical or numerical methods, because of the discontinuities at the interfaces between different materials. The simple solution method of separation of variables, in the case of a constant coefficient $\alpha(x)$, is no longer practical. Instead, analytical solutions can be found using the classical layer potential [1, 2], though not in closed form. Monte Carlo methods have also been employed [3, 4, 5, 6, 7]. In $[8,9]$ it is shown how a closed-form representation of the resolvent kernel can be obtained for problems such as those considered in this paper, from which a fundamental solution can be obtained via inversion of a Laplace transform. However, for such problems, this inversion must be carried out numerically.

Numerical methods, such as finite element and finite difference methods $[10,11]$, have difficulties with PDEs of this form because the discontinuities of the coefficient introduce discontinuities in the first or second derivatives of the solution, which implies slower decay of its Fourier coefficients [12]. As a result, higher spatial resolution is required to represent the solution, and therefore time-stepping methods are susceptible to stiffness. For both implicit and explicit time-stepping methods, stiffness leads to a substantial increase 
in computational expense, due to the CFL constraint forcing a smaller time step for explicit methods, or ill-conditioning in systems solved by implicit methods that slows the convergence of iterative methods.

Both (2) and (3) can be written in the form

$$
u_{t}+L u=0 .
$$

The operator $L$ is either $\alpha(x)^{2} \partial^{2} / \partial x^{2}$ or $-\partial / \partial x\left(\alpha(x)^{2} \partial / \partial x\right)$ for (2) or (3), respectively. When the eigenvalues $\left\{\lambda_{j}\right\}_{j=0}^{\infty}$ and eigenfunctions $\left\{V_{j}\right\}_{j=0}^{\infty}$ of $L$ are known, separation of variables easily yields a solution of (5) that has the form

$$
u(x, t)=\sum_{j=0}^{\infty} V_{j}(x) e^{-\lambda_{j} t}\left\langle W_{j}, f\right\rangle /\left\langle W_{j}, V_{j}\right\rangle,
$$

where $u(x, 0)=f(x)$ is the initial condition, and $\langle\cdot, \cdot\rangle$ denotes the standard inner product of square integrable functions on $(0,2 \pi)$,

$$
\langle f, g\rangle=\int_{0}^{2 \pi} \overline{f(x)} g(x) d x .
$$

The eigenfunction of the adjoint $L^{*}$ corresponding to $\lambda_{j}$ is $W_{j}(x)$ (for (3), $W_{j}=V_{j}$ ). Not only is a solution of this form readily computed, thus avoiding most of the pitfalls of numerical methods (with the exception of rounding error and discretization error in arising from the inner products), but the form of the solution is far more conducive to qualitative analysis of its behavior than numerical solutions that simply produce a vector of values of the solution at grid points.

Unfortunately, a solution of the form (6) can only be obtained in special cases such as a constant coefficient, for which the eigensystem of $L$ is known. We will still use separation of variables, but because our coefficients are not simply constant, there will be a significant challenge in computing the eigenfunctions and eigenvalues. In this paper, we present a method for accurately and efficiently computing these eigenvalues and eigenfunctions in the case of a piecewise constant coefficient. The resulting algorithm for computing the eigenvalues and coefficients of the eigenfunctions is highly parallelizable, with computational expense independent of spatial resolution. From this algorithm, we can obtain a solution in a much more useful form, and more efficiently, than what is produced by conventional numerical methods. It will also be seen that the proposed solution method is more scalable than such numerical methods. 
This work builds on previous work in [13], for the self-adjoint problem (3) in the case of $n=2$; that is, only a single interface between two materials. The solution of the corresponding eigenvalue problem, for the case of homogeneous Dirichlet boundary conditions, was presented in [14], but without a numerical procedure for practical computation. In this paper, we consider periodic boundary conditions, but the algorithm can readily be modified for the cases of homogeneous Dirichlet and Neumann boundary conditions [13].

The outline of the paper is as follows. In Section 2 we explain how the $S A K$ principle can be used to obtain estimates of eigenvalues, which can serve as initial guesses for an iterative method to compute eigenvalues with high accuracy. In Section 3 we derive equations that characterize the eigenvalues, and explain how the eigenfunctions can be constructed. Numerical results are presented in Section 4, and conclusions are given in Section 5.

\section{Applying the $S A K$ Principle}

For the two cases (self-adjoint and non-self adjoint) considered in this paper, with periodic boundary conditions, we will develop a practical algorithm for computing highly accurate approximate eigenvalues and eigenfunctions. This algorithm involves solving a nonlinear equation for each eigenvalue by iteration, which requires an initial guess.

We first discuss how an accurate initial guess can be obtained from the Uncertainty Principle, and its interpretation by Fefferman in [15]. The Uncertainty Principle indicates that the position and momentum of a particle cannot both be measured with arbitrarily high accuracy [16]. This is mathematically equivalent to stating that a function $f$ and its Fourier transform $\hat{f}$ cannot be concentrated within an arbitrarily small box in phase space, which is the Cartesian product of physical space and frequency space $[15,17,18]$. Consider the self-adjoint differential operator

$$
A(x, D)=\sum_{|\alpha| \leq m} a_{\alpha}(x) D^{\alpha}, \quad D=\frac{1}{i} \frac{\partial}{\partial x},
$$

where $i=\sqrt{-1}$ is the imaginary unit. The symbol of $A(x, D)$ is defined by

$$
A(x, \omega)=e^{-i \omega x} A(x, D) e^{i \omega x}=\sum_{|\alpha| \leq m} a_{\alpha}(x) \omega^{\alpha} .
$$


The $S A K$ principle, due to Fefferman [15], states that the number of eigenvalues of $A(x, D)$ that are less than $K$ is approximately equal to the number of "distorted unit cubes" that can be packed in the set $S(A, K)=$ $\{(x, \omega) \mid A(x, \omega)<K\}$. By applying $A(x, D)$ to a function $\phi(x)$ which is concentrated within a distorted unit cube in phase space, centered at $\left(x_{0}, \omega_{0}\right)$, we have

$$
A(x, D) \phi(x) \approx A\left(x_{0}, \omega_{0}\right) \phi(x)
$$

where $A\left(x_{0}, \omega_{0}\right)$ is an approximate eigenvalue, and $\phi(x)$ is an approximate eigenfunction. Intuitively, $\phi(x)$ is a more accurate approximate eigenfunction if $A(x, \omega)$ does not vary much within the region in which $\phi(x)$ is concentrated. Therefore, we seek eigenfunctions concentrated in subsets of phase space that are derived from the level curves of $A(x, \omega)$.

If we define

$$
A(x, D)=-\frac{\partial}{\partial x} \alpha^{2}(x) \frac{\partial}{\partial x}=D \alpha^{2}(x) D
$$

then

$$
A(x, \omega)=\left\{\begin{array}{cl}
\alpha_{1}^{2} \omega^{2}, & 0 \leq x<2 \pi \rho_{1} \\
\alpha_{2}^{2} \omega^{2}, & 2 \pi \rho_{1} \leq x<2 \pi \rho_{2} \\
\vdots & \\
\alpha_{n}^{2} \omega^{2}, & 2 \pi \rho_{n-1} \leq x<2 \pi .
\end{array}\right.
$$

The level curves of this symbol are defined by

$$
\left\{(x, \omega) \mid \omega=\omega_{i}, 2 \pi \rho_{i-1} \leq x<2 \pi \rho_{i}, i=1,2, \ldots, n\right\}
$$

where, for convenience, we define $\rho_{0}=0$ and $\rho_{n}=1$, and the values $\omega_{i}$, $i=1, \ldots, n$, satisfy

$$
\alpha_{1} \omega_{1}=\cdots=\alpha_{n} \omega_{n} .
$$

To estimate which level curves can be used to define regions of phase in which eigenfunctions are approximately concentrated, we use a heuristic introduced in [19], that the eigenvalues of $A(x, D)$ are approximately characterized by decomposing phase space into regions bounded by level curves of $A(x, \omega)$ such that the area of each region is $2 \pi$.

In the following example with $n=4$ pieces, we will demonstrate how the eigenvalues are estimated. We will choose $\omega$ values $\left(\omega_{11}, \omega_{12}, \omega_{13}, \omega_{14}\right)$ to represent our frequency values of the eigenfunction corresponding to the smallest nonzero eigenvalue $\lambda_{1}$. By examining the level curves of $A(x, \omega)$, the values of $\omega$ are all related by the equations

$$
\alpha_{1} \omega_{11}=\alpha_{2} \omega_{12}=\alpha_{3} \omega_{13}=\alpha_{4} \omega_{14}
$$




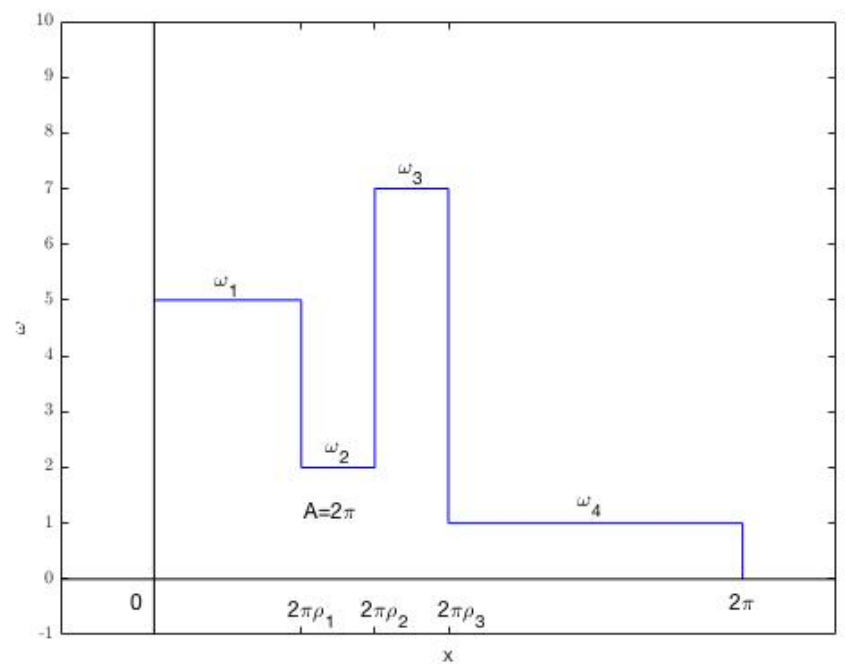

Figure 1: The region in phase space for the eigenfunction.

Figure 1 shows the visual representation of how the $S A K$ principle can help us approximate the values of $\omega_{1 i}$, where $i=1,2,3,4$. The area of the polygon in the figure is

$$
\omega_{11} 2 \pi \rho_{1}+\omega_{12}\left(2 \pi \rho_{2}-2 \pi \rho_{1}\right)+\omega_{13}\left(2 \pi \rho_{3}-2 \pi \rho_{2}\right)+\omega_{14}\left(2 \pi-2 \pi \rho_{3}\right)=2 \pi .
$$

For the case where $\alpha_{1}=1, \alpha_{2}=2, \alpha_{3}, \alpha_{4}=4, \rho_{1}=\frac{3}{8}, \rho_{2}=\frac{1}{2}$, and $\rho_{3}=\frac{3}{4}$ along with the relationship between the $\alpha_{i}$ and $\omega_{j i}$ values established by the $S A K$ Principle, the equation simplifies to

$$
2 \pi=4 \omega_{14} \frac{3 \pi}{4}+2 \omega_{14} \frac{\pi}{4}+\frac{4}{3} \omega_{14} \frac{\pi}{2}+\omega_{14} \frac{\pi}{2}
$$

which has the solution

$$
\omega_{14}=\frac{3}{7} \text {. }
$$

We conclude that our initial guess for $\omega_{j 4}$, to obtain eigenvalues $\lambda_{j}, j=$ $1,2, \ldots$, should be integer multiples of $\frac{3}{7}$. Heuristically, we expect each pair of exact frequencies that are the closest to $\omega_{j n}$ to fall within the interval $\left(\omega_{(j-1) n}, \omega_{(j+1) n}\right)$, where we define $\omega_{0 n}=0$. Once the frequencies $\omega_{j i}$ are obtained, the eigenvalues are given by

$$
\lambda_{j}=\left(\alpha_{n} \omega_{j n}\right)^{2} .
$$


Once the eigenvalues are computed, the corresponding eigenfunctions can be constructed, as will be discussed in detail in the next section.

In the case of the general piecewise constant coefficient $\alpha(x)$ defined in (1), we have

$$
2 \pi=\sum_{i=1}^{n} \omega_{j i} 2 \pi\left(\rho_{i}-\rho_{i-1}\right), \quad \rho_{0}=0, \quad \rho_{n}=1 .
$$

If we define $\tau_{i}=\frac{\alpha_{n}}{\alpha_{i}}$, then we have $\omega_{j i}=\tau_{i} \omega_{j n}$, and (10) reduces to

$$
2 \pi=\sum_{i=1}^{n} \tau_{i} \omega_{j n} 2 \pi\left(\rho_{i}-\rho_{i-1}\right) .
$$

Then we can solve for the value of the initial guess $\omega_{j n}^{(0)}$ that will be used for iteration:

$$
\omega_{j n}^{(0)}=\left\lceil\frac{j}{2}\right\rceil \frac{1}{\sum_{i=1}^{n} \tau_{i}\left(\rho_{i}-\rho_{i-1}\right)},
$$

where $\lceil x\rceil$ is the "ceiling function" (that is, the smallest integer greater than or equal to $x)$. This is because each integer multiple of $\left(\sum_{i=1}^{n} \tau_{i}\left(\rho_{i}-\rho_{i-1}\right)\right)^{-1}$ is an estimate of a pair of nonzero eigenvalues.

\section{Computing Eigenvalues and Eigenfunctions}

We seek eigenfunctions of the form

$$
V_{j}(x)=\left\{\begin{array}{cc}
a_{j 1} \cos \left(\omega_{j 1} x\right)+b_{j 1} \sin \left(\omega_{j 1} x\right) & 0 \leq x<2 \pi \rho_{1} \\
a_{j 2} \cos \left(\omega_{j 2} x\right)+b_{j 2} \sin \left(\omega_{j 2} x\right) & 2 \pi \rho_{1} \leq x<2 \pi \rho_{2} \\
\vdots & \\
a_{j n} \cos \left(\omega_{j n} x\right)+b_{j n} \sin \left(\omega_{j n} x\right) & 2 \pi \rho_{n-1} \leq x<2 \pi
\end{array}\right.
$$

where $\omega_{j 1}, \ldots, \omega_{j n}$ are the frequencies, $a_{j 1}, \ldots, a_{j n}$ and $b_{j 1}, \ldots, b_{j n}$ represent the unknown amplitudes and phase shifts, $\rho_{i}$ represents where the interface between materials occurrs, and the index value $n$ represents how many pieces are being described. 


\subsection{Non-Self-adjoint Case}

We first consider the non-self-adjoint operator $A(x, D)=\alpha^{2}(x) D^{2}$. The eigenfunctions for the piecewise constant coefficient case will be described by equation (13). Our goal is to eliminate all of the parameters except for $\omega_{j n}$ so we can solve for it, therefore obtaining the eigenvalues.

The restrictions and conditions of $2 \pi$-periodicity and continuity at the interface between materials leads us to the elimination of $a_{j 1}$ and $b_{j 1}$. For the two-piece case, we have the following conditions [20]:

$V_{j}(x)$ and its derivative must be $2 \pi$-periodic:

$$
\begin{aligned}
& V_{j 1}(0)=V_{j 2}(2 \pi) \\
& V_{j 1}^{\prime}(0)=V_{j 2}^{\prime}(2 \pi)
\end{aligned}
$$

In addition, $V_{j}(x)$ and its derivative must be continuous at the interface:

$$
\begin{aligned}
& V_{j 1}\left(2 \pi \rho_{1}\right)=V_{j 2}\left(2 \pi \rho_{1}\right) \\
& V_{j 1}^{\prime}\left(2 \pi \rho_{1}\right)=V_{j 2}^{\prime}\left(2 \pi \rho_{1}\right)
\end{aligned}
$$

From the first periodicity condition (14), it follows that

$$
a_{j 1}=a_{j 2} \cos \left(2 \pi \omega_{j 2}\right)+b_{j 2} \sin \left(2 \pi \omega_{j 2}\right) .
$$

The periodicity of the derivative from (15) yields an equation for $b_{j 1}$ :

$$
b_{j 1}=\frac{-a_{j 2} \omega_{j 2} \sin \left(2 \pi \omega_{j 2}\right)+b_{j 2} \omega_{j 2} \cos \left(2 \pi \omega_{j 2}\right)}{\omega_{j 1}} .
$$

The continuity conditions (16), (17) give us the equations

$$
\begin{aligned}
a_{j 1} \cos \left(2 \pi \rho \omega_{j 1}\right)+b_{j 1} \sin \left(2 \pi \rho \omega_{j 1}\right)= & a_{j 2} \cos \left(2 \pi \rho \omega_{j 2}\right)+ \\
& b_{j 2} \sin \left(2 \pi \rho \omega_{j 2}\right) \\
-a_{j 1} \omega_{j 1} \sin \left(2 \pi \rho \omega_{j 1}\right)+b_{j 1} \omega_{j 1} \cos \left(2 \pi \rho \omega_{j 1}\right)= & -a_{j 2} \omega_{j 2} \sin \left(2 \pi \rho \omega_{j 2}\right)+ \\
& b_{j 2} \omega_{j 2} \cos \left(2 \pi \rho \omega_{j 2}\right) .
\end{aligned}
$$

The equations (18), (19) are substituted into (20) and (21) to eliminate $a_{j 1}$ and $b_{j 1}$. Then, we let $\tau_{1}=\frac{\alpha_{2}}{\alpha_{1}}$ so that $\omega_{j 1}=\tau_{1} \omega_{j 2}$, so that $\omega_{j 1}$ is eliminated from (20) and (21) as well. This yields the homogeneous system of linear equations

$$
\left(\left[\begin{array}{ll}
u_{11} & u_{12} \\
u_{21} & u_{22}
\end{array}\right]+\left[\begin{array}{ll}
d_{11} & d_{12} \\
d_{21} & d_{22}
\end{array}\right]\right)\left[\begin{array}{l}
a_{j 2} \\
b_{j 2}
\end{array}\right]=\left[\begin{array}{l}
0 \\
0
\end{array}\right]
$$


where

$$
\begin{aligned}
& u_{11}=c_{22} c_{11}-\frac{1}{\tau_{1} \omega_{j 2}} \omega_{j 2} s_{22} s_{11} \\
& u_{12}=s_{22} c_{11}+\frac{1}{\tau_{1} \omega_{j 2}} \omega_{j 2} c_{22} s_{11} \\
& u_{21}=-\tau_{1} \omega_{j 2} c_{22} s_{11}-\frac{1}{\tau_{1} \omega_{j 2}} \omega_{j 2} s_{22} \tau_{1} \omega_{j 2} c_{11} \\
& u_{22}=-\tau_{1} \omega_{j 2} s_{22} s_{11}+\frac{1}{\tau_{1} \omega_{j 2}} \omega_{j 2} c_{22} \tau_{1} \omega_{j 2} c_{11},
\end{aligned}
$$

and

$$
d_{11}=-c_{12}, \quad d_{12}=-s_{12}, \quad d_{21}=\omega_{j 2} s_{12}, \quad d_{22}=-\omega_{j 2} c_{12},
$$

with $c_{i k}=\cos \left(2 \pi \rho_{i} \tau_{k} \omega_{j n}\right)$ and $s_{i k}=\sin \left(2 \pi \rho_{i} \tau_{k} \omega_{j n}\right)$. This is the matrix-vector equation for the two-piece case. The basic idea is to extend this to the $n$ piece case so we can construct a numerical method that will solve the matrix vector equation (22).

The four-piece case can be handled using the same procedure. The conditions based on periodicity and continuity that are similar to Equations (14), (15), (16), and (17) allow us to eliminate parameters and then obtain the system

$$
\left[\begin{array}{cccccc}
-c_{12} & -s_{12} & 0 & 0 & u_{11} & u_{12} \\
\omega_{j 2} s_{12} & -\omega_{j 2} c_{12} & 0 & 0 & u_{21} & u_{22} \\
c_{22} & s_{22} & -c_{23} & -s_{23} & 0 & 0 \\
-\omega_{j 2} s_{12} & \omega_{j 2} s_{12} & \omega_{j 3} s_{33} & -\omega_{j 3} c_{33} & 0 & 0 \\
0 & 0 & c_{33} & s_{33} & -c_{34} & -s_{34} \\
0 & 0 & -\omega_{j 3} s_{33} & \omega_{j 3} s_{33} & \omega_{j 4} s_{34} & -\omega_{j 4} s_{34}
\end{array}\right]\left[\begin{array}{c}
a_{j 2} \\
b_{j 2} \\
a_{j 3} \\
b_{j 3} \\
a_{j 4} \\
b_{j 4}
\end{array}\right]=\left[\begin{array}{l}
0 \\
0 \\
0 \\
0 \\
0 \\
0
\end{array}\right]
$$

where

$$
\begin{gathered}
u_{11}=c_{44} c_{11}-\frac{1}{\tau_{1} \omega_{j 4}} \omega_{j 4} s_{44} s_{11}, \quad u_{12}=s_{44} c_{11}+\frac{1}{\tau_{1} \omega_{j 4}} \omega_{j 4} c_{44} s_{11}, \\
u_{21}=-\tau_{1} \omega_{j 4} c_{44} s_{11}-\frac{1}{\tau_{1} \omega_{j 4}} \tau_{1} \omega_{j 4}^{2} s_{44} c_{11}, \quad u_{22}=-\tau_{1} \omega_{j 4} s_{44} s_{11}+\frac{1}{\tau_{1} \omega_{j 4}} \tau_{1} \omega_{j 4}^{2} c_{44} c_{11} .
\end{gathered}
$$

For the general case of $n$ pieces, we obtain the system

$$
M \mathbf{y}=\mathbf{0}
$$


which has the form

$$
\left[\begin{array}{ccccc}
D_{1} & & & & U_{0} \\
F_{1} & D_{2} & & & \\
& F_{2} & D_{3} & & \\
& & \ddots & \ddots & \\
& & & F_{n-2} & D_{n-1}
\end{array}\right]\left[\begin{array}{c}
a_{j 2} \\
b_{j 2} \\
\vdots \\
a_{j n} \\
b_{j n}
\end{array}\right]=\left[\begin{array}{c}
0 \\
\vdots \\
\vdots \\
0
\end{array}\right]
$$

The matrix $M$ is a block lower triangular matrix plus a rank two-update, with block entries $D_{1}, \ldots, D_{n-1}, F_{1}, \ldots, F_{n-2}$ and $U_{0}$ defined by

$$
\begin{gathered}
D_{i}=\left[\begin{array}{cc}
-c_{i, i+1} & -s_{i, i+1} \\
\omega_{j, i+1} s_{i, i+1} & -\omega_{j, i+1} c_{i, i+1}
\end{array}\right], \\
F_{i}=\left[\begin{array}{cc}
c_{i+1, i+1} & s_{i+1, i+1} \\
-\omega_{j, i+1} s_{i+1, i+1} & \omega_{j, i+1} c_{i+1, i+1}
\end{array}\right], \\
U_{0}=\left[\begin{array}{cc}
c_{n n} c_{11}-\frac{\omega_{j n} s_{n n} s_{11}}{\tau_{1} \omega_{j n}} & s_{n n} c_{11}+\frac{\omega_{j n} c_{n n} s_{11}}{\tau_{1} \omega_{j n}} \\
-\tau_{1} \omega_{j n} c_{n n} s_{11}-\frac{\tau_{1} \omega_{j n}^{2} s_{n n} c_{11}}{\tau_{1} \omega_{j n}} & -\tau_{1} \omega_{j n} s_{n n} s_{11}+\frac{\tau_{1} \omega_{j n}^{2} c_{n n} c_{11}}{\tau_{1} \omega_{j n}}
\end{array}\right],
\end{gathered}
$$

where we again use the notation $c_{i k}=\cos \left(2 \pi \rho_{i} \tau_{k} \omega_{j n}\right)$ and $s_{i k}=\sin \left(2 \pi \rho_{i} \tau_{k} \omega_{j n}\right)$.

The matrix $M$ can be written as

$$
M=M_{0}+U X^{T}, \quad U=\left[\begin{array}{c}
U_{0} \\
0
\end{array}\right], \quad X=\left[\begin{array}{c}
0 \\
I_{2}
\end{array}\right]
$$

where $I_{2}$ is a $2 \times 2$ identity matrix. The vector $\mathbf{y}$ is made up of the unknown coefficients of our eigenfunctions except the initial $a_{j 1}$ and $b_{j 1}$ that were found earlier using periodicity conditions:

$$
\mathbf{y}=\left[\begin{array}{c}
a_{j 2} \\
b_{j 2} \\
\vdots \\
a_{j n} \\
b_{j n}
\end{array}\right]
$$

In order to obtain $\mathbf{y} \in \operatorname{ker}(M)$, we need to find values of $\omega_{j n}$ that make $\operatorname{det}(M)=0$.

From the Matrix Determinant Lemma, we have

$$
\operatorname{det}(M)=\operatorname{det}\left(D_{1}\right) \operatorname{det}\left(D_{2}\right) \ldots \operatorname{det}\left(D_{n-1}\right) \operatorname{det}\left(I_{2}+X^{T} Z\right), \quad M_{0} Z=U
$$


Because $M_{0}$ is block lower bidiagonal, with $2 \times 2$ blocks, $Z$ is easily computed in $O(n)$ arithmetic operations using block forward substitution. $M_{0}$ is invertible, as the determinant of the $2 \times 2 D_{i}$ blocks are nonzero; specifically, $\operatorname{det} D_{i}=\omega_{i+1}$. This allows us to focus on the last determinant, of $I_{2}+X^{T} Z$. This determinant is then passed through the secant method, with initial guesses obtained using the $S A K$ principle as described in Section 2, to find the values of $\omega_{j n}$ that make $\operatorname{det}(M)=0$.

Once $M$ is made singular, the system (23) can be rewritten as

$$
M_{0} \mathbf{y}+U X^{T} \mathbf{y}=\mathbf{0}
$$

Then, after multiplying by $M_{0}^{-1}$, which is known to exist, we have

$$
\mathbf{y}+Z X^{T} \mathbf{y}=\mathbf{0}
$$

and we can multiply both sides by $X^{T}$, which yields the equation

$$
X^{T} \mathbf{y}+X^{T} Z X^{T} \mathbf{y}=\mathbf{0} .
$$

We can then factor out $X^{T} \mathbf{y}$ to obtain

$$
\left(I_{2}+X^{T} Z\right)\left(X^{T} \mathbf{y}\right)=\mathbf{0} .
$$

If we define $R=I_{2}+X^{T} Z$ and $\mathbf{g}=X^{T} \mathbf{y}$, the system (31) reduces to

$$
R \mathrm{~g}=\mathbf{0}
$$

If we let

$$
R=\left[\begin{array}{ll}
a & b \\
c & d
\end{array}\right]
$$

then we have solutions

$$
\mathbf{g}_{1}=\left[\begin{array}{c}
b \\
-a
\end{array}\right], \quad \mathbf{g}_{2}=\left[\begin{array}{c}
d \\
-c
\end{array}\right]
$$

We then choose

$$
\mathbf{g}=\left[\begin{array}{l}
a_{j n} \\
b_{j n}
\end{array}\right]
$$

to be the larger of $\mathbf{g}_{1}$ and $\mathbf{g}_{2}$ in magnitude, which can be measured using, for example, the $\ell_{2}$ norm on $\mathbb{R}^{2}$ defined as $\sqrt{\left(a_{j n}\right)^{2}+\left(b_{j n}\right)^{2}}$, to avoid difficulties 
caused by roundoff error. Now that we have $a_{j n}$ and $b_{j n}$, we can substitute $X^{T} \mathbf{y}=\mathbf{g}$ into equation (30) to obtain

$$
M_{0} \mathbf{y}+U \mathbf{g}=\mathbf{0}
$$

which has the unique solution

$$
\mathbf{y}=-Z \mathbf{g}
$$

From (18) and (19) that came from the periodicity conditions, we obtain $a_{j 1}$ and $b_{j 1}$. Now that we have solved for all of the coefficients as well as the frequencies $\omega_{j i}, i=1,2, \ldots, n$, we can construct the eigenfunctions of our differential operator. The following theorem and its proof describe this construction.

Theorem 1. Let $\omega_{j n}$ be such that the matrix $M$ from (23) is singular. Let $\mathbf{y}$ be defined as in (28) and computed as in (33), where $Z$ is as defined in (29) and $\mathbf{g}$ is a nontrivial solution of (32), where $R=I_{2}+X^{T} Z$ and $X$ is as defined in (27). Furthermore, let $a_{j 1}$ and $b_{j 1}$ be given by

$$
\begin{aligned}
a_{j 1} & =a_{j n} \cos \left(2 \pi \omega_{j n}\right)+b_{j n} \sin \left(2 \pi \omega_{j n}\right), \\
b_{j 1} & =\frac{-a_{j n} \omega_{j n} \sin \left(2 \pi \omega_{j n}\right)+b_{j n} \omega_{j n} \cos \left(2 \pi \omega_{j n}\right)}{\omega_{j 1}} .
\end{aligned}
$$

Finally, let $\omega_{j 1}, \ldots, \omega_{j n}$ satisfy (8). Then $V_{j}(x)$, as defined in (13), is a $C^{1}(\mathbb{R}) 2 \pi$-periodic eigenfunction of the operator $L=-\alpha(x)^{2} \partial^{2} / \partial x^{2}$, where $\alpha(x)$ is as defined in (1), with corresponding eigenvalue $\lambda_{n}=\left(\alpha_{n} \omega_{j n}\right)^{2}$.

Proof: In view of (29), because $\operatorname{det} M=0$ and the blocks $D_{1}, \ldots, D_{n-1}$ are nonsingular, it follows that $R$ is singular so (32) has a nontrivial solution g. We then have

$$
\begin{aligned}
M \mathbf{y} & =M_{0} \mathbf{y}+U X^{T} \mathbf{y} \\
& =-M_{0} Z \mathbf{g}-U X^{T} Z \mathbf{g} \\
& =-U \mathbf{g}-U X^{T} Z \mathbf{g} \\
& =-U\left(I_{2}+X^{T} Z\right) \mathbf{g} \\
& =-U R \mathbf{g} \\
& =\mathbf{0} .
\end{aligned}
$$


We now consider $V_{j}(x)$ from (13), with its coefficients $a_{j 2}, b_{j 2}, \ldots, a_{j n}, b_{j n}$ taken from the entries of $\mathbf{y}$ and (34), (35). From (34), (35), both $V_{j}(x)$ and $V_{j}^{\prime}(x)$ are $2 \pi$-periodic. From the equations

$$
F_{i}\left[\begin{array}{l}
a_{j, i+1} \\
b_{j, i+1}
\end{array}\right]+D_{i+1}\left[\begin{array}{l}
a_{j, i+2} \\
b_{j, i+2}
\end{array}\right]=\mathbf{0}, \quad i=1,2, \ldots, n-2,
$$

and from (24), (25), we obtain, for $i=1,2, \ldots, n-2$,

$$
\begin{aligned}
a_{j, i+1} \cos \left(2 \pi \rho_{i+1} \omega_{j, i+1}\right)+ & \\
b_{j, i+1} \sin \left(2 \pi \rho_{i+1} \omega_{j, i+1}\right)= & a_{j, i+2} \cos \left(2 \pi \rho_{i+1} \omega_{j, i+2}\right)+ \\
& b_{j, i+2} \sin \left(2 \pi \rho_{i+1} \omega_{j, i+2}\right), \\
-a_{j, i+1} \omega_{j, i+1} \sin \left(2 \pi \rho_{i+1} \omega_{j, i+1}\right)+ & \\
b_{j, i+1} \omega_{j, i+1} \cos \left(2 \pi \rho_{i+1} \omega_{j, i+1}\right)= & -a_{j, i+2} \omega_{j, i+2} \sin \left(2 \pi \rho_{i+1} \omega_{j, i+2}\right)+ \\
& b_{j, i+2} \omega_{j, i+2} \cos \left(2 \pi \rho_{i+1} \omega_{j, i+2}\right),
\end{aligned}
$$

from which we can conclude that $V_{j}(x)$ and $V_{j}^{\prime}(x)$ are continuous at $x=$ $2 \pi \rho_{2}, \ldots, 2 \pi \rho_{n-1}$. Similarly, from

$$
D_{1}\left[\begin{array}{l}
a_{j 2} \\
b_{j 2}
\end{array}\right]+u_{0}\left[\begin{array}{l}
a_{j n} \\
b_{j n}
\end{array}\right]=\mathbf{0}
$$

we can establish continuity of $V_{j}(x)$ and $V_{j}^{\prime}(x)$ at $x=2 \pi \rho_{1}$ as well. To see this, we note that

$$
u_{0}\left[\begin{array}{l}
a_{j n} \\
b_{j n}
\end{array}\right]=F_{0}\left[\begin{array}{l}
a_{j 1} \\
b_{j 1}
\end{array}\right],
$$

as a consequence of (34), (35). Combined with periodicity, we have that $V_{j}(x)$ and $V_{j}^{\prime}(x)$ are continuous on the entire real line. Finally, to establish that $V_{j}(x)$ is an eigenfunction, we have, on $\left[2 \pi \rho_{i-1}, 2 \pi \rho_{i}\right)$,

$$
L V_{j}(x)=\alpha_{i}^{2} \omega_{j i}^{2}\left[a_{j i} \cos \left(\omega_{j i} x\right)+b_{j i} \sin \left(\omega_{j i} x\right)\right]=\left(\alpha_{n} \omega_{j n}\right)^{2} V_{j}(x),
$$

in view of $(8)$.

\subsection{Self-adjoint Case}

For the self-adjoint operator $A(x, D)=D \alpha^{2}(x) D$, we again define each eigenfunction $V_{j}(x), j=1,2, \ldots$, as in (13). The frequencies $\left\{\omega_{j i}\right\}_{i=1}^{n}$ are related by

$$
\begin{array}{r}
\alpha_{1} \omega_{j 1}=\cdots=\alpha_{n} \omega_{j n} \\
\tau_{i}=\frac{\alpha_{n}}{\alpha_{i}}, \quad \omega_{j i}=\tau_{1} \omega_{i n}, \quad i=1, \ldots, n-1 .
\end{array}
$$


By substituting $\omega_{j i}=\tau_{i} \omega_{j n}$ we have

$$
\begin{aligned}
a_{1} \cos \left(\tau_{1} \omega_{j n} x\right)+b_{1} \sin \left(\tau_{1} \omega_{j n} x\right) & =V_{j 1}(x) \\
a_{2} \cos \left(\tau_{2} \omega_{j n} x\right)+b_{2} \sin \left(\tau_{2} \omega_{j n} x\right) & =V_{j 2}(x) \\
& \vdots \\
a_{n-1} \cos \left(\tau_{n-1} \omega_{j n} x\right)+b_{n-1} \sin \left(\tau_{n} \omega_{j n} x\right) & =V_{j, n-1}(x) \\
a_{n} \cos \left(\tau_{n} \omega_{j n} x\right)+b_{n} \sin \left(\tau_{n} \omega_{j n} x\right) & =V_{j n}(x)
\end{aligned}
$$

By applying the periodicity condition $V_{j}(0)=V_{j}(2 \pi)$, we can write

$$
a_{1}=a_{n} \cos \left(\tau_{n} \omega_{j n} 2 \pi\right)+b_{n} \sin \left(\tau_{n} \omega_{j n} 2 \pi\right)
$$

Enforcing continuity at the interfaces,

$$
V_{j i}\left(2 \pi \rho_{i}\right)=V_{j, i+1}\left(2 \pi \rho_{i}\right), \quad i=1,2, \ldots, i-1,
$$

yields the conditions

$$
\begin{array}{rll}
a_{1} \cos \left(\tau_{1} \omega_{j n} 2 \pi \rho_{1}\right)+b_{1} \sin \left(\tau_{1} \omega_{j n} 2 \pi \rho_{1}\right)= & a_{2} \cos \left(\tau_{2} \omega_{j n} 2 \pi \rho_{1}\right)+b_{2} \sin \left(\tau_{2} \omega_{j n} 2 \pi \rho_{1}\right) \\
a_{2} \cos \left(\tau_{2} \omega_{j n} 2 \pi \rho_{2}\right)+b_{2} \sin \left(\tau_{2} \omega_{j n} 2 \pi \rho_{2}\right)= & a_{3} \cos \left(\tau_{3} \omega_{j n} 2 \pi \rho_{2}\right)+b_{3} \sin \left(\tau_{3} \omega_{j n} 2 \pi \rho_{2}\right) \\
\vdots & \\
a_{n-2} \cos \left(\tau_{n-2} \omega_{j n} 2 \pi \rho_{n-2}\right)+ & \\
b_{n-2} \sin \left(\tau_{n-2} \omega_{j n} 2 \pi \rho_{n-2}\right)= & a_{n-1} \cos \left(\tau_{n-1} \omega_{j n} 2 \pi \rho_{n-2}\right)+ \\
& b_{n-1} \sin \left(\tau_{n-1} \omega_{j n} 2 \pi \rho_{n-2}\right) \\
a_{n-1} \cos \left(\tau_{n-1} \omega_{j n} 2 \pi \rho_{n-1}\right)+= & \\
b_{n-1} \sin \left(\tau_{n-1} \omega_{j n} 2 \pi \rho_{n-1}\right)= & a_{n} \cos \left(\tau_{n} \omega_{j n} 2 \pi \rho_{n-1}\right)+ \\
& & b_{n} \sin \left(\tau_{n} \omega_{j n} 2 \pi \rho_{n-1}\right) .
\end{array}
$$

From the matching of conormal derivatives (see $[14,13]$ ),

$$
\alpha_{i}^{2} V_{j i}^{\prime}\left(2 \pi \rho_{i}\right)=\alpha_{i+1}^{2} V_{j, i+1}^{\prime}\left(2 \pi \rho_{i}\right), \quad i=1,2, \ldots, n-1,
$$


we obtain the conditions

$$
\begin{aligned}
&-a_{1} \sin \left(\tau_{1} \omega_{j n} 2 \pi \rho_{1}\right)+b_{1} \cos \left(\tau_{1} \omega_{j n} 2 \pi \rho_{1}\right)= \frac{\tau_{1}}{\tau_{2}}\left(-a_{2} \sin \left(\tau_{2} \omega_{j n} 2 \pi \rho_{1}\right)+b_{2} \cos \left(\tau_{2} \omega_{j n} 2 \pi \rho_{1}\right)\right) \\
&-a_{2} \sin \left(\tau_{2} \omega_{j n} 2 \pi \rho_{2}\right)+b_{2} \cos \left(\tau_{2} \omega_{j n} 2 \pi \rho_{2}\right)= \frac{\tau_{2}}{\tau_{3}}\left(-a_{3} \sin \left(\tau_{3} \omega_{j n} 2 \pi \rho_{2}\right)+b_{3} \cos \left(\tau_{3} \omega_{j n} 2 \pi \rho_{2}\right)\right) \\
& \vdots \\
&-a_{n-2} \sin \left(\tau_{n-2} \omega_{j n} 2 \pi \rho_{n-2}\right)+ \\
& b_{n-2} \cos \left(\tau_{n-2} \omega_{j n} 2 \pi \rho_{n-2}\right)=\frac{\tau_{n-2}}{\tau_{n-1}}\left(-a_{n-1} \sin \left(\tau_{n-1} \omega_{j n} 2 \pi \rho_{n-2}\right)+\right. \\
&-a_{n-1} \sin \left(\tau_{n-1} \omega_{j n} 2 \pi \rho_{n-1}\right)+ \\
& b_{n-1} \cos \left(\tau_{n-1} \omega_{j n} 2 \pi \rho_{n-1}\right)=\left.\frac{\tau_{n-1}\left(-a_{n} \sin \left(\tau_{n} \omega_{j n} 2 \pi \rho_{n-1}\right)+\right.}{\tau_{n}}\left(\tau_{n-1} 2 \pi \rho_{n-2}\right)\right) \\
&\left.b_{n} \cos \left(\tau_{n} \omega_{j n} 2 \pi \rho_{n-1}\right)\right)
\end{aligned}
$$

Let $V_{0}(x)$ denote the eigenfunction corresponding to $\lambda_{0}=0$, so $V_{0}(x) \equiv 1$. From the orthogonality of the eigenfunctions, we have $\left\langle V_{j}, V_{0}\right\rangle=0$, or

$$
\begin{gathered}
\int_{0}^{2 \pi \rho_{1}}\left[a_{1} \cos \left(\tau_{1} \omega_{j n} x\right)+b_{1} \sin \left(\tau_{1} \omega_{j n} x\right)\right] d x+ \\
\int_{2 \pi \rho_{1}}^{2 \pi \rho_{2}}\left[a_{2} \cos \left(\tau_{2} \omega_{j n} x\right)+b_{2} \sin \left(\tau_{2} \omega_{j n} x\right)\right] d x+ \\
\cdots+\int_{2 \pi \rho_{n-2}}^{2 \pi \rho_{n-1}}\left[a_{n-1} \cos \left(\tau_{n-1} \omega_{j n} x\right)+b_{n-1} \sin \left(\tau_{n-1} \omega_{j n} x\right)\right] d x+ \\
\int_{2 \pi \rho_{n-1}}^{2 \pi}\left[a_{n} \cos \left(\tau_{n} \omega_{j n} x\right)+b_{n} \sin \left(\tau_{n} \omega_{j n} x\right)\right] d x=0 .
\end{gathered}
$$

After applying all of the above conditions, we have $2 n$ constraints that form the homogeneous system $M \mathbf{y}=\mathbf{0}$, where

$$
M=\left[\begin{array}{cccccc}
D_{1} & & & & & F_{1} \\
F_{2} & D_{2} & & & & \\
& \ddots & \ddots & & & \\
& & \ddots & \ddots & & \\
& & & \ddots & \ddots & \\
E_{1} & \ldots & \ldots & E_{n-2} & F_{n} & D_{n}
\end{array}\right], \quad \mathbf{y}=\left[\begin{array}{c}
a_{2} \\
b_{2} \\
\vdots \\
a_{n} \\
b_{n} \\
a_{1} \\
b_{1}
\end{array}\right]
$$


The block entries have the form

$$
\begin{aligned}
D_{i} & =\left[\begin{array}{cc}
-c_{i+1, i} & -s_{i+1, i} \\
\frac{\tau_{i} s_{i+1, i}}{\tau_{i+1}} & -\frac{\tau_{i} c_{i+1, i}}{\tau_{i+1}}
\end{array}\right], \quad i=1, \ldots, n-1, \\
D_{n} & =\left[\begin{array}{cc}
s_{11} & \left(-c_{11}+1\right) \\
-1 & 0
\end{array}\right], \\
F_{i} & =\left[\begin{array}{cc}
c_{i, i} & s_{i, i} \\
-s_{i, i} & c_{i, i}
\end{array}\right], \quad i=1, \ldots, n-1, \\
F_{n} & =\left[\begin{array}{cc}
\frac{s_{n, n}-s_{n, n-1}}{\omega_{j n} \tau_{n}} & \frac{-c_{n, n}+c_{n, n-1}}{\omega_{j n} \tau_{n}} \\
c_{n, n} & s_{n, n}
\end{array}\right], \\
E_{i} & =\left[\begin{array}{cc}
\frac{s_{n, n}-s_{n, n-1}}{\omega_{j n} \tau_{n}} & \frac{-c_{n, n}+c_{n, n-1}}{\omega_{j n} \tau_{n}} \\
0 & 0
\end{array}\right], \quad i=1, \ldots, n-2,
\end{aligned}
$$

where, as before, $c_{i k}=\cos \left(2 \pi \rho_{i} \tau_{k} \omega_{j n}\right)$ and $s_{i k}=\sin \left(2 \pi \rho_{i} \tau_{k} \omega_{j n}\right)=s_{i k}$.

From the Matrix Determinant Lemma, we have

$\operatorname{det}(M)=\operatorname{det}\left(M_{0}\right) \operatorname{det}\left(I_{2}+X^{T} M_{0}^{-1} U\right)=\left(\prod_{i=1}^{n} \operatorname{det}\left(D_{i}\right)\right) \operatorname{det}\left(I_{2}+X^{T} M_{0}^{-1} U\right)$

where

$$
M_{0}^{-1} U=Z=\left[\begin{array}{c}
Z_{1} \\
Z_{2} \\
\vdots \\
Z_{n}
\end{array}\right], \quad U=\left[\begin{array}{c}
F_{1} \\
0 \\
\vdots \\
0
\end{array}\right], \quad X^{T}=\left[\begin{array}{llll}
0 & \ldots & 0 & I_{2}
\end{array}\right],
$$

and block forward substitution yields

$$
\begin{aligned}
Z_{1} & =D_{1}^{-1} F_{1} \\
Z_{2} & =-D_{2}^{-1} F_{2} D_{1}^{-1} F_{1} \\
Z_{3} & =D_{3}^{-1} F_{3} D_{2}^{-1} F_{2} D_{1}^{-1} F_{1} \\
& \vdots \\
Z_{n} & =-D_{n}^{-1}\left(\sum_{i=1}^{n-2} E_{i} Z_{i}+F_{n} Z_{n-1}\right)
\end{aligned}
$$


Proceeding as in the non-self-adjoint case, we can solve $M \mathbf{y}=\mathbf{0}$ by first solving

$$
\left(I_{2}+X^{T} Z\right) \mathbf{g}=\mathbf{0}, \quad \mathbf{g}=X^{T} \mathbf{y}=\left[\begin{array}{l}
a_{1} \\
b_{1}
\end{array}\right]
$$

and then solving $M_{0} \mathbf{y}=-U \mathbf{g}$ which yields

$$
\mathbf{y}=-Z \mathbf{g}=\left[\begin{array}{c}
a_{2} \\
b_{2} \\
\vdots \\
a_{n} \\
b_{n} \\
\mathbf{z}
\end{array}\right]
$$

We are now able to construct the eigenfunctions. The correctness of these eigenfunctions can be verified in the same way as in the proof of Theorem 1.

\subsection{Solving for $\omega_{j n}$ by Iteration}

Using the estimate of $\omega_{j n}$ suggested by the $S A K$ principle as described in Section 2, we can solve the equation

$$
\operatorname{det}\left(I_{2}+X^{T} M_{0}^{-1} U\right)=0
$$

for $\omega_{j n}$ by iteration, using this estimate as an initial guess. For this purpose, we use the secant method, as it is rapidly convergent and does not require the evaluation of derivatives.

Let $\omega_{j n}^{(0)}$ be the estimate obtained via the $S A K$ principle. For our second initial guess that is required by the secant method, we simply perturb $\omega_{j n}^{(0)}$. We also "safeguard" the iteration by requiring all iterates to fall within the interval $\left|\omega-\omega_{j n}^{(0)}\right|<\omega_{1 n}^{(0)}$. We remind the reader that each $\omega_{j n}^{(0)}$ is an integer multiple of $\omega_{1 n}^{(0)}$. If an iterate generated by the secant method falls outside this interval, we reject it, and substitute an alternative iterate as follows:

- If there has been at least one positive value and one negative value of the determinant, then we perform one iteration of bisection, and update the search interval accordingly.

- If all iterates produce determinants of the same sign, then we set our new iterate equal to the midpoint of the largest interval between iterates. 
Generally, for $j$ odd, $\omega_{j n}^{(0)}$ is near the exact values of $\omega_{j n}$ and $\omega_{j+1, n}$, and in most cases, it has been observed to lie between these values. Therefore, after obtaining $\omega_{j n}$ by iteration, we adjust the initial guess $\omega_{j+1, n}^{(0)}$ as follows:

$$
\omega_{j+1, n}^{(0)}=2 \omega_{j n}^{(0)}-\omega_{j n} .
$$

We also reduce the search interval by half, according to whether $\omega_{j n}$ is less than or greater than $\omega_{j n}^{(0)}$. For example, in the case $\omega_{j n}<\omega_{j n}^{(0)}$, we search for $\omega_{j+1, n}$ in the interval $\left(\omega_{j n}^{(0)}, \omega_{j n}^{(0)}+\omega_{1 n}^{(0)}\right)$. If the secant iteration fails, then we search in the interval $\left(\omega_{j n}^{(0)}-\omega_{1 n}^{(0)}, \omega_{j n}\right)$.

\subsection{Algorithm Description}

We now describe, in pseudocode, the algorithms for computing the eigenvalues and eigenfunctions, and from there, the solution of a PDE with given initial data. We first consider the non-self-adjoint case. The following algorithm describes the computation of $\omega_{2 j-1, n}$ and $\omega_{2 j, n}$ corresponding to a pair of nonzero eigenvalues $\lambda_{2 j-1}, \lambda_{2 j}$. The vectors $\vec{\tau}$ and $\vec{\rho}$ consist of the values $\tau_{1}, \ldots, \tau_{n}$ and $\rho_{1}, \ldots, \rho_{n-1}$, respectively.

function $\left[\omega_{2 j-1, n}, \omega_{2 j, n}\right]=\operatorname{getpair}(\vec{\tau}, \vec{\rho}, j)$

Obtain $\omega_{2 j-1, n}^{(0)}$ as in (12)

Solve $\operatorname{det}(R)=0$ for $\omega_{2 j-1, n}$ using iteration

$R=I_{2}+X^{T} Z$, defined as in Section 3.1

Use $\omega_{2 j-1, n}^{(0)}$ and a small perturbation as initial guesses

$\omega_{2 j, n}^{(0)}=2 \omega_{2 j-1, n}^{(0)}-\omega_{2 j-1, n}$

Solve $\operatorname{det}(R)=0$ for $\omega_{2 j, n}$ using iteration

Use $\omega_{2 j, n}^{(0)}$ and a small perturbation as initial guesses

We now describe the overall process, that relies on the getpair function. We denote by $\mathbf{x}$ a vector of equally spaced grid points in $[0,2 \pi)$, that is partitioned into vectors $\mathbf{x}_{1}, \mathbf{x}_{2}, \ldots, \mathbf{x}_{n}$ such that the elements of $\mathbf{x}_{i}$ belong to $\left[2 \pi \rho_{i-1}, 2 \pi \rho_{i}\right)$ for $i=1,2, \ldots, n$. The initial data $f(x)$ is described by a vector $\mathbf{f}$ consisting of the values of $f(x)$ at the grid points in $\mathbf{x}$. The output of this algorithm is a vector $\mathbf{u}$ that contains an approximation of the solution $u(x, t)$ of $(2)$ on $(0,2 \pi)$, evaluated at the grid points in $\mathbf{x}$, for given $t$.

$\mathbf{v}_{0}=\left[\begin{array}{lll}1 & \cdots & 1\end{array}\right]^{T} \in \mathbb{R}^{n}$

$\mathbf{w}_{0}=1 / \alpha(\mathbf{x})^{2}$ 
$\mathbf{u}=\mathbf{v}_{0} \mathbf{v}_{0}^{T} \mathbf{f} / \mathbf{w}_{0}^{T} \mathbf{v}_{0}$

for $j=1,2, \ldots$ do

$\left[\omega_{2 j-1, n}, \omega_{2 j, n}\right]=\operatorname{getpair}(\vec{\tau}, \vec{\rho}, j)$

for $i=2 j-1,2 j$ do

$\lambda_{i}=\alpha_{n}^{2} \omega_{i, n}^{2}$

Form $M_{0}, U$ and $X$ as in (27)

Solve $M_{0} Z=U$ using block forward substitution

$R=I_{2}+X^{T} Z$

$P=\left[\begin{array}{cc}0 & -1 \\ 1 & 0\end{array}\right]$

$G=P R^{T}$

Let $\mathrm{g}$ be the column of $G$ with largest $\ell_{2}$-norm

Solve $M_{0} \mathbf{y}=-U \mathbf{g}$ using block forward substitution

for $k=2, \ldots, n$ do

$$
\begin{aligned}
& a_{k}=y_{2 k-3} \\
& b_{k}=y_{2 k-2}
\end{aligned}
$$

\section{end for}

$$
\begin{aligned}
& a_{1}=a_{n} \cos \left(2 \pi \omega_{i n}\right)+b_{n} \sin \left(2 \pi \omega_{i n}\right) \\
& b_{1}=\left(-a_{n} \omega_{i n} \sin \left(2 \pi \omega_{i n}\right)+b_{n} \omega_{i n} \cos \left(2 \pi \omega_{i n}\right)\right) /\left(\tau_{1} \omega_{i n}\right) \\
& \mathbf{v}_{1}=a_{1} \cos \left(\omega_{i 1} \mathbf{x}_{1}\right)+b_{k} \sin \left(\omega_{i 1} \mathbf{x}_{1}\right) \\
& \text { for } k=2, \ldots, n \mathbf{d o} \\
& \quad \mathbf{v}_{i}=\left[\begin{array}{c}
a_{k} \cos \left(\omega_{i k} \mathbf{x}_{k}\right)+b_{k} \sin \left(\omega_{i k} \mathbf{x}_{k}\right)
\end{array}\right]
\end{aligned}
$$$$
\text { end for }
$$ \\ end for}

$\mathbf{w}_{j}=\mathbf{v}_{j} / \alpha(\mathbf{x})^{2}$

end for

$$
\mathbf{u}=\mathbf{u}+e^{-\lambda_{i} t} \mathbf{v}_{i} \mathbf{w}_{i}^{T} \mathbf{f} / \mathbf{w}_{i}^{T} \mathbf{v}_{i}
$$

\section{end for}

The outermost loop terminates when the terms added to $\mathbf{u}$ are smaller than a chosen tolerance.

We now describe the algorithm for the self-adjoint case, which is similar. In fact, the function getpair is no different; it simply works with the equation $\operatorname{det}(M)=0$, where $M$ is as defined in (36). The overall process for solving (3) with given initial data $f(x)$, represented by the vector $\mathbf{f}$, and time $t$ is as follows.

$\mathbf{u}=\operatorname{mean}(\mathbf{f})$ 


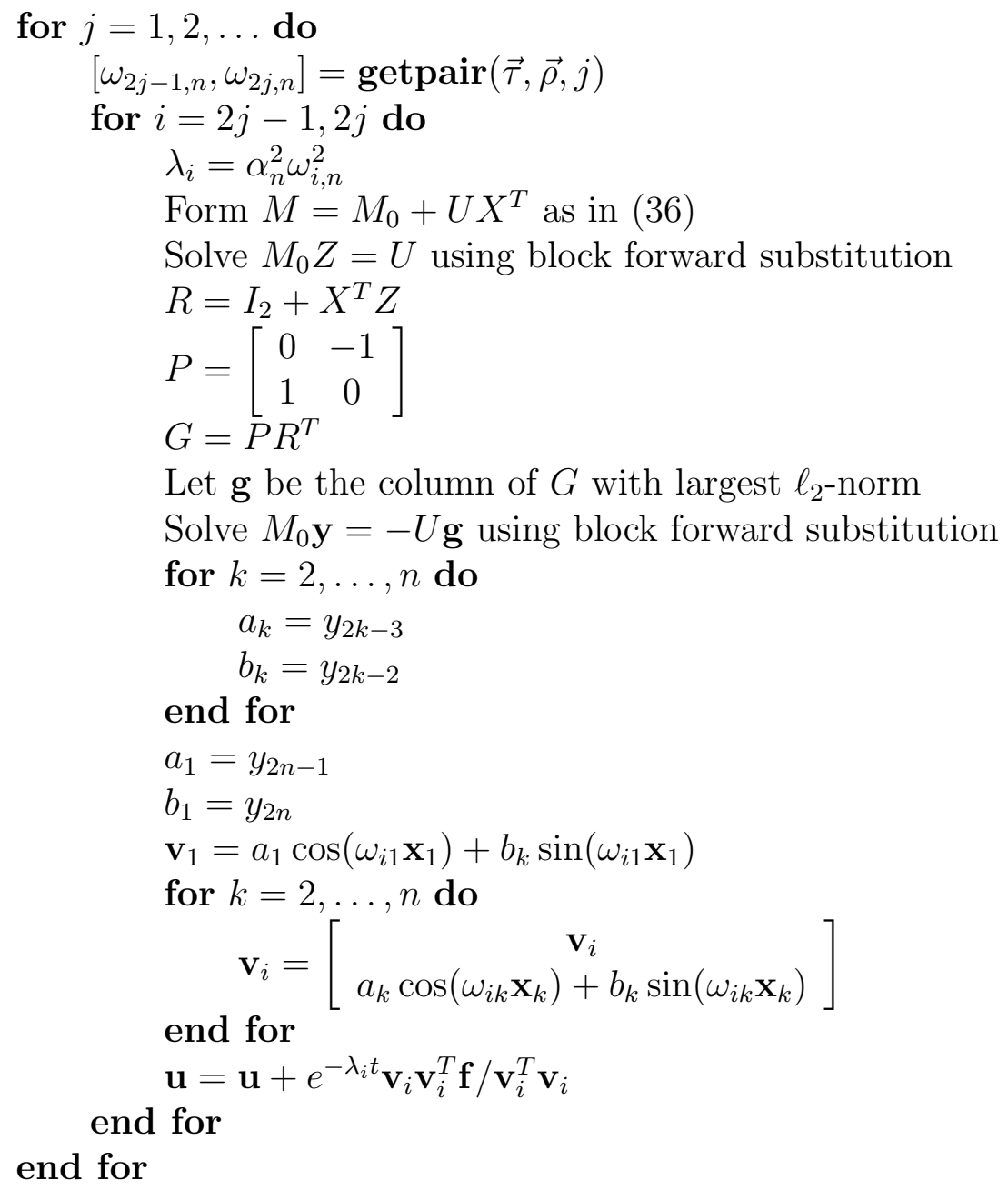

\section{Numerical Results}

In this section we demonstrate the effectiveness of our approach for computing eigenvalues and eigenfunctions of the spatial differential operators under consideration, and also using them to solve the diffusion equations (2) and (3). We will compare the computed eigenvalues to those obtained using the MatLaB function eigs on a sparse matrix $A$ that discretizes each operator by finite differences in the non-self-adjoint case, and finite elements with piecewise linear basis functions, and elements of equal width, in the self-adjoint case. 
When solving PDEs, we compare our approach, labeled "eig-exp" in tables, to the following methods, all of which use the appropriate matrix $A$ :

- Crank-Nicolson (labeled "C-N" in tables),

- Approximating $e^{-A t} \mathbf{u}(0)$ using rational Krylov projection, as in [21] (labeled "Krylov" in tables), and

- The Matlab ODE solver ode15s (labeled "ode15s" in tables).

We apply these methods with different grid sizes $N=1024,2048,4096$ to examine scalability and spatial convergence. To measure accuracy, solutions are compared to a reference solution computed using ode15s with a larger number of grid points, $N_{\text {ref }}=16,384$, and the smallest allowed time step.

We calculate the error $\mathcal{E}_{N}$ in each computed solution $\tilde{\mathbf{u}}^{N}$ with respect to the reference solution $\mathbf{u}^{N_{\text {ref }}}$ by the $\ell_{\infty}$ norm of the difference between the computed solution and the restriction of the reference solution to the $N$-point grid; that is,

$$
\mathcal{E}_{N}=\max _{1 \leq i \leq N}\left|u_{m(i)}^{N_{\mathrm{ref}}}-\tilde{u}_{i}^{N}\right|
$$

where $m(i)=\frac{N_{\text {ref }}}{N}(i-1)+1$ is used to map the values of $\mathbf{u}^{N}$ on its $N$-point

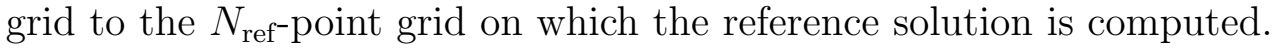

The value of $N_{\text {ref }}$ was the largest power of 2 such that MATLAB could compute solutions via ode15s for all cases without exceeding storage limits. We note that each reference solution required, at minimum, approximately 30 seconds (for $t_{f}=0.01$ ) to several minutes (for $t_{f}=1$ ) to compute, which, as will be seen in this section, is not competitive with any of the methods featured.

\subsection{Non-Self-adjoint Case}

\subsubsection{Computing Eigenvalues}

Tables 1-4 show the smallest ten nonzero eigenvalues of the operator $-\alpha^{2}(x) \partial_{x}^{2}$, as computed using the $S A K$ principle with secant iteration, as in Section 3.1, and using the MATLAB function eigs with a $N \times N$ matrix obtained via finite-difference discretization. In Tables 1 and $3, N=2^{12}$, and in Tables 2 and $4, N=2^{23}$. Tables 1 and 2 feature a coefficient with $n=4$ pieces, while $n=7$ in Tables 3 and 4 .

The number of iterations of the secant method is reported in each table. The relative error shown in these tables is the relative difference between the 
two sets of computed eigenvalues. The error in the eigenvalues obtained by secant iteration arises only from roundoff error and the termination of the iteration, which occurs when the absolute difference between iterates is less than $10^{-8}$. As such, the error given in the tables is actually indicative of the error in the eigenvalues computed by eigs. By comparing the results for the two grid sizes, it can be seen that these eigenvalues are generally converging to the ones obtained via secant iteration.

\begin{tabular}{rrrrr}
\hline$j$ & $\lambda_{j}(S A K)$ & $\lambda_{j}($ eig $)$ & iterations & relative error \\
\hline 1 & 2.213728201966723 & 2.214546835371368 & 8 & $3.70 \mathrm{e}-04$ \\
2 & 3.908115974058471 & 3.911393874896230 & 6 & $8.39 \mathrm{e}-04$ \\
3 & 10.082518215776597 & 10.094371927238504 & 8 & $1.18 \mathrm{e}-03$ \\
4 & 13.388169744247897 & 13.388419936929154 & 5 & $1.87 \mathrm{e}-05$ \\
5 & 24.341673078716386 & 24.282295735999661 & 8 & $2.44 \mathrm{e}-03$ \\
6 & 28.324126848500523 & 28.372268962285855 & 6 & $1.70 \mathrm{e}-03$ \\
7 & 45.780880112858526 & 45.788474347323032 & 8 & $1.66 \mathrm{e}-04$ \\
8 & 48.879344768381820 & 48.851440781676736 & 7 & $5.71 \mathrm{e}-04$ \\
9 & 68.937479644237115 & 68.960230443978261 & 7 & $3.30 \mathrm{e}-04$ \\
10 & 78.422800516160351 & 78.488178032528552 & 5 & $8.34 \mathrm{e}-04$ \\
\hline
\end{tabular}

Table 1: Eigenvalues computed using the $S A K$ principle with secant iteration, and eigs on a finite-difference matrix, of the operator $-\alpha^{2}(x) \partial_{x}^{2}$ with $\vec{\alpha}=[1,2,3,4], \vec{\rho}=[3 / 8,1 / 2,3 / 4]$ and $N=4,096$ grid points.

\begin{tabular}{rrrrr}
\hline$j$ & $\lambda_{j}(S A K)$ & $\lambda_{j}($ eig $)$ & iterations & relative error \\
\hline 1 & 2.213728201966723 & 2.213728529834787 & 8 & $1.48 \mathrm{e}-07$ \\
2 & 3.908115974058471 & 3.908111184822544 & 6 & $1.23 \mathrm{e}-06$ \\
3 & 10.082518215776597 & 10.082519780918508 & 8 & $1.55 \mathrm{e}-07$ \\
4 & 13.388169744247897 & 13.388165032603471 & 5 & $3.52 \mathrm{e}-07$ \\
5 & 24.341673078716386 & 24.341667699188690 & 8 & $2.21 \mathrm{e}-07$ \\
6 & 28.324126848500523 & 28.324127431593521 & 6 & $2.06 \mathrm{e}-08$ \\
7 & 45.780880112858526 & 45.780880262752021 & 8 & $3.27 \mathrm{e}-09$ \\
8 & 48.879344768381820 & 48.879180460807362 & 7 & $3.36 \mathrm{e}-06$ \\
9 & 68.937479644237115 & 68.937529509562850 & 7 & $7.23 \mathrm{e}-07$ \\
10 & 78.422800516160351 & 78.422819299731756 & 5 & $2.40 \mathrm{e}-07$ \\
\hline
\end{tabular}

Table 2: Eigenvalues computed using the $S A K$ principle with secant iteration, and eigs on a finite-difference matrix, of the operator $-\alpha^{2}(x) \partial_{x}^{2}$ with $\vec{\alpha}=[1,2,3,4], \vec{\rho}=[3 / 8,1 / 2,3 / 4]$ and $N=8,388,608$ grid points.

\subsubsection{Solving PDEs}

Tables 5-16 compare the performance of the methods listed at the beginning of this section for solving the heat equation (2) at $t=t_{f}$, in terms of efficiency (odd-numbered tables) and accuracy (even-numbered tables). We use smooth initial data $u(x, 0)=\sin 2 x$ or a smoothed characteristic function 


\begin{tabular}{rrrrr}
\hline$j$ & $\lambda_{j}(S A K)$ & $\lambda_{j}(\mathrm{eig})$ & iterations & relative err \\
\hline 1 & 2.208768644228804 & 2.209651291736346 & 8 & $4.00 \mathrm{e}-04$ \\
2 & 3.698554293240147 & 3.702052427217607 & 6 & $9.45 \mathrm{e}-04$ \\
3 & 9.835635137659720 & 9.828492009057010 & 7 & $7.26 \mathrm{e}-04$ \\
4 & 12.720325761465526 & 12.729268908769621 & 5 & $7.03 \mathrm{e}-04$ \\
5 & 22.868339996850548 & 22.875773648650320 & 7 & $3.25 \mathrm{e}-04$ \\
6 & 27.588004958504420 & 27.599415204504702 & 6 & $4.14 \mathrm{e}-04$ \\
7 & 43.421531530822222 & 43.457994198681568 & 6 & $8.40 \mathrm{e}-04$ \\
8 & 46.394983148646915 & 46.396792705599310 & 9 & $3.90 \mathrm{e}-05$ \\
9 & 66.148542025384060 & 66.108839948075811 & 8 & $6.00 \mathrm{e}-04$ \\
10 & 78.425134748368009 & 78.184966066576692 & 5 & $3.06 \mathrm{e}-03$ \\
\hline
\end{tabular}

Table 3: Eigenvalues computed using the $S A K$ principle with secant iteration, and eigs on a finite-difference matrix, of the operator $-\alpha^{2}(x) \partial_{x}^{2}$ with $\vec{\alpha}=[1,2,3,4,2,6,4], \vec{\rho}=$ $[3 / 8,1 / 2,3 / 4,4 / 5,5 / 6,6 / 7]$ and $N=4,096$ grid points.

\begin{tabular}{rrrrr}
\hline$j$ & $\lambda_{j}(S A K)$ & $\lambda_{j}($ eig $)$ & iterations & relative err \\
\hline 1 & 2.208768644228804 & 2.208769184319859 & 8 & $2.45 \mathrm{e}-07$ \\
2 & 3.698554293240147 & 3.698550379791000 & 6 & $1.06 \mathrm{e}-06$ \\
3 & 9.835635137659720 & 9.835640922799071 & 7 & $5.88 \mathrm{e}-07$ \\
4 & 12.720325761465526 & 12.720321404686686 & 5 & $3.43 \mathrm{e}-07$ \\
5 & 22.868339996850548 & 22.868328754223263 & 7 & $4.92 \mathrm{e}-07$ \\
6 & 27.588004958504420 & 27.588006625416060 & 5 & $6.04 \mathrm{e}-08$ \\
7 & 43.421531530822222 & 43.421536596326689 & 6 & $1.17 \mathrm{e}-07$ \\
8 & 46.394983148646915 & 46.394956202557971 & 9 & $5.81 \mathrm{e}-07$ \\
9 & 66.148542025384060 & 66.148534991216707 & 8 & $1.06 \mathrm{e}-07$ \\
10 & 78.425134748368009 & 78.425179055166083 & 5 & $5.65 \mathrm{e}-07$ \\
\hline
\end{tabular}

Table 4: Eigenvalues computed using the $S A K$ principle with secant iteration, and eigs on a finite-difference matrix, of the operator $-\alpha^{2}(x) \partial_{x}^{2}$ with $\vec{\alpha}=[1,2,3,4,2,6,4], \vec{\rho}=$ $[3 / 8,1 / 2,3 / 4,4 / 5,5 / 6,6 / 7]$ and $N=8,388,608$ grid points.

that still has steep jumps,

$$
u(x, 0)=\left(\frac{1}{\pi} \tan ^{-1}\left(d\left(x-c_{1}\right)\right)+\frac{\pi}{2}\right)-\left(\frac{1}{\pi} \tan ^{-1}\left(d\left(x-c_{2}\right)\right)+\frac{\pi}{2}\right),
$$

with $c_{1}=3 \pi / 4, c_{2}=5 \pi / 4$ and $d=40$, the graph of which is shown in Figure 2. The piecewise constant coefficient $\alpha^{2}(x)$ consists of either four, seven or eight pieces. The three choices of $\alpha(x)$ featured in these experiments are shown in Figure 3.

The various methods used are configured as follows:

- For eigenfunction expansion, we compute eigenvalues $\lambda_{0}, \lambda_{1}, \ldots, \lambda_{m}$ and eigenfunctions $V_{0}(x), V_{1}(x), \ldots, V_{m}(x)$ using the algorithm described in Section 3.1, where $m$ is chosen so that $e^{-\lambda_{j} t_{f}}<\epsilon$ for $j>m$ and $\epsilon=10^{-4}$. Then, the approximate solution is obtained from the eigen- 


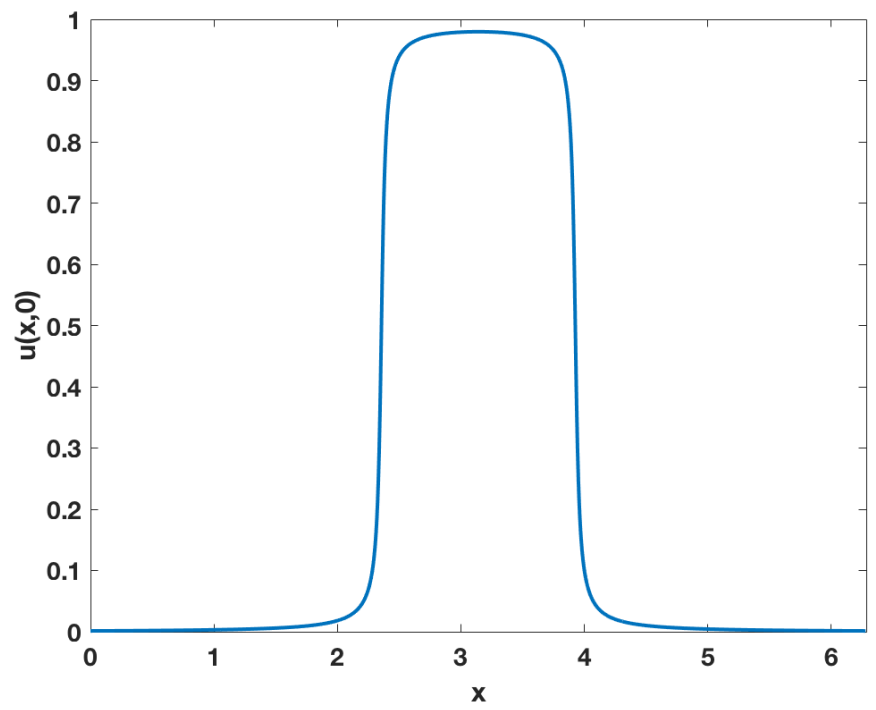

Figure 2: Smoothed characteristic function used as initial data $u(x, 0)$

function expansion

$$
u(x, t) \approx \sum_{j=0}^{m} V_{j}(x) e^{-\lambda_{j} t} \frac{\left\langle W_{j}, u(\cdot, 0)\right\rangle}{\left\langle W_{j}, V_{j}\right\rangle}
$$

where $W_{j}(x)=V_{j}(x) / \alpha^{2}(x)$ is the eigenfunction of the adjoint operator $-\left(\alpha^{2}(x) \partial_{x}^{2}\right)^{*}$ corresponding to $\lambda_{j}$ and $\langle\cdot, \cdot\rangle$ is the standard inner product on $[0,2 \pi]$.

- Crank-Nicolson performs 100 time steps of length $t_{f} / 100$.

- Krylov projection produces iterates $\mathbf{u}_{1}, \ldots, \mathbf{u}_{p}$, such that $\left\|\mathbf{u}_{p}-\mathbf{u}_{p-1}\right\|_{\infty}<$ $10^{-4}\left\|\mathbf{u}_{p}\right\|_{\infty}$.

- The value $\Delta t_{\text {avg }}$ reported in the tables is the average time step length used by ode15s to obtain a solution that satisfies its default error tolerance.

It can be seen that generally, our method computes the solution at time $t_{f}$ with greater efficiency than the other methods, with rational Krylov projection being the closest competitor. It is worth noting that as the number 


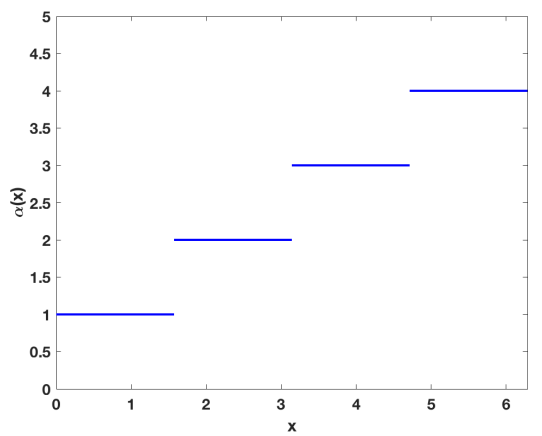

(a)

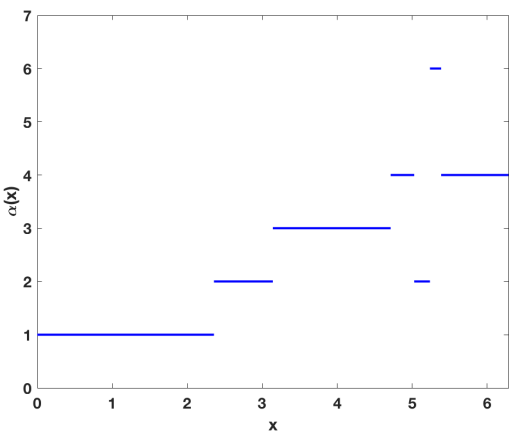

(b)

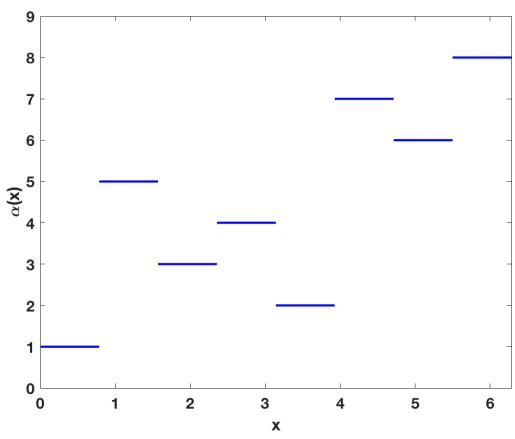

(c)

Figure 3: Choices of $\alpha(x)$ used in numerical experiments, with four (upper left plot), seven (upper right plot) and eight (lower plot) pieces.

of grid points $N$ increases, the execution time increases more substantially for all of the other methods, compared to eigenfunction expansion. That is, eigenfunction expansion is more scalable. This is shown in Figure 4, which displays the execution times from Table 7 to show that not only is the time taken by our method less, but it also grows at a slower rate as $N$ increases.

Furthermore, our method is much more parallelizable, as all of the eigenpairs can be computed independently of one another, whereas the other methods featured do not lend themselves to parallelism. For our method, such parallelism was not included in the results presented in this paper. Figures 5-10 show the solutions computed by the different methods. We see that, visually, the solutions computed by all four methods match one another, just as the tables indicate that the error in these solutions is generally small.

Regarding accuracy, we see that generally, our approach lags slightly be- 
hind the other methods for $t_{f}=0.01$, but catches up to or surpasses them as $t_{f}$ increases. We also note that in most cases, all four methods exhibit roughly first-order convergence in space. An exception is Crank-Nicolson in the case of the initial data (37), because the sharp jumps in the initial data cause deterioration in accuracy of finite differences. This deterioration is not exhibited by Krylov projection or ode15s, that also use finite differences, due to adaptivity. For these three methods that use finite differences, second-order accuracy in space is the norm, but not expected in this case due to the eigenfunctions having a discontinuous second derivative. The spatial accuracy of our approach will be discussed further at the end of this section.

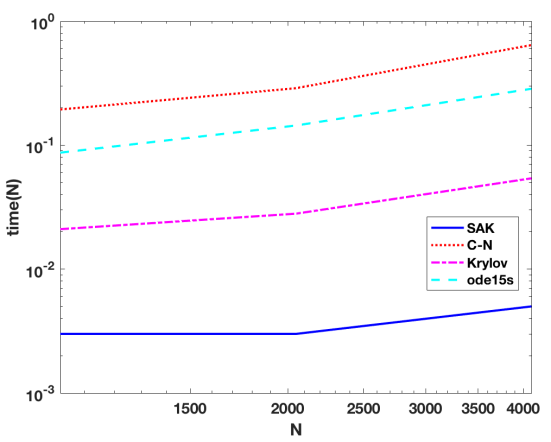

(a)

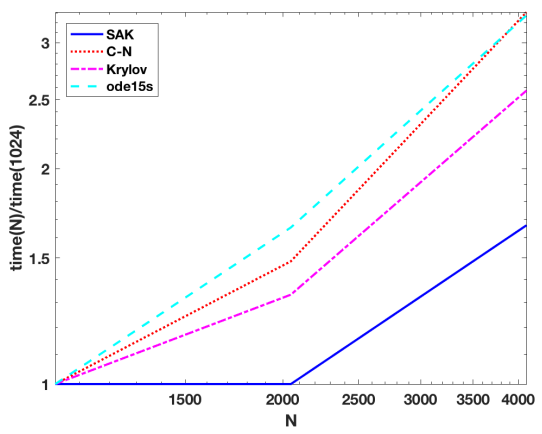

(b)

Figure 4: Left plot: execution time as a function of grid size $N$ for eigenfunction expansion, Crank-Nicolson, rational Krylov projection, and ode15s. Right plot: execution time for all methods normalized to display growth as a function of $N$.

\begin{tabular}{|l|c|c|c|c|c|c|c|c|}
\hline \multicolumn{2}{|c|}{} & \multicolumn{2}{c|}{ eig-exp } & C-N & \multicolumn{2}{c|}{ Krylov } & \multicolumn{2}{c|}{ ode15s } \\
\hline$t_{f}$ & $N$ & time & $m$ & time & time & $p$ & time & $\Delta t_{\text {avg }}$ \\
\hline 0.01 & 1024 & 0.019 & 25 & 0.132 & 0.138 & 13 & 0.041 & $2.703 \mathrm{e}-04$ \\
& 2048 & 0.018 & 25 & 0.274 & 0.065 & 13 & 0.091 & $2.564 \mathrm{e}-04$ \\
& 4096 & 0.028 & 25 & 0.987 & 0.107 & 13 & 0.113 & $2.439 \mathrm{e}-04$ \\
\hline 0.1 & 1024 & 0.008 & 11 & 0.125 & 0.038 & 10 & 0.052 & $1.538 \mathrm{e}-03$ \\
& 2048 & 0.009 & 11 & 0.246 & 0.049 & 10 & 0.101 & $1.493 \mathrm{e}-03$ \\
& 4096 & 0.012 & 11 & 0.597 & 0.072 & 10 & 0.204 & $1.429 \mathrm{e}-03$ \\
\hline 1 & 1024 & 0.004 & 5 & 0.126 & 0.018 & 7 & 0.084 & $9.709 \mathrm{e}-03$ \\
& 2048 & 0.004 & 5 & 0.254 & 0.025 & 7 & 0.132 & $9.524 \mathrm{e}-03$ \\
& 4096 & 0.007 & 5 & 0.644 & 0.056 & 7 & 0.259 & $9.259 \mathrm{e}-03$ \\
\hline
\end{tabular}

Table 5: Execution times (in seconds) from Table 7 for methods listed from left to right: eigenfunction expansion with $m$ terms, Crank-Nicolson, rational Krylov projection with $p$ projection steps, and ode15s with average time step $\Delta t_{\text {avg }}$, for solving (2) to time $t_{f}$ on a $N$-point uniform grid with $u(x, 0)=\sin 2 x, \vec{\alpha}=[1,2,3,4]$ and $\vec{\rho}=[1 / 4,1 / 2,3 / 4]$. 


\begin{tabular}{|l|c|c|c|c|c|c|c|c|}
\hline \multicolumn{2}{|c|}{} & \multicolumn{2}{c|}{ eig-exp } & C-N & \multicolumn{2}{c|}{ Krylov } & \multicolumn{2}{c|}{ ode15s } \\
\hline$t_{f}$ & $N$ & error & $m$ & error & error & $p$ & error & $\Delta t_{\text {avg }}$ \\
\hline 0.01 & 1024 & $7.5 \mathrm{e}-05$ & 25 & $1.5 \mathrm{e}-03$ & $1.5 \mathrm{e}-03$ & 13 & $1.5 \mathrm{e}-03$ & $2.703 \mathrm{e}-04$ \\
& 2048 & $9.3 \mathrm{e}-05$ & 25 & $7.0 \mathrm{e}-04$ & $6.8 \mathrm{e}-04$ & 13 & $6.9 \mathrm{e}-04$ & $2.564 \mathrm{e}-04$ \\
& 4096 & $9.8 \mathrm{e}-05$ & 25 & $3.0 \mathrm{e}-04$ & $2.9 \mathrm{e}-04$ & 13 & $3.0 \mathrm{e}-04$ & $2.439 \mathrm{e}-04$ \\
\hline 0.1 & 1024 & $1.5 \mathrm{e}-04$ & 11 & $2.4 \mathrm{e}-03$ & $2.4 \mathrm{e}-03$ & 10 & $2.4 \mathrm{e}-03$ & $1.538 \mathrm{e}-03$ \\
& 2048 & $1.6 \mathrm{e}-04$ & 11 & $1.1 \mathrm{e}-03$ & $1.1 \mathrm{e}-03$ & 10 & $1.1 \mathrm{e}-03$ & $1.493 \mathrm{e}-03$ \\
& 4096 & $1.6 \mathrm{e}-04$ & 11 & $4.7 \mathrm{e}-04$ & $4.8 \mathrm{e}-04$ & 10 & $4.8 \mathrm{e}-04$ & $1.429 \mathrm{e}-03$ \\
\hline 1 & 1024 & $9.1 \mathrm{e}-06$ & 5 & $9.8 \mathrm{e}-05$ & $9.4 \mathrm{e}-05$ & 7 & $1.0 \mathrm{e}-04$ & $9.709 \mathrm{e}-03$ \\
& 2048 & $5.8 \mathrm{e}-06$ & 5 & $4.7 \mathrm{e}-05$ & $4.3 \mathrm{e}-05$ & 7 & $7.7 \mathrm{e}-05$ & $9.524 \mathrm{e}-03$ \\
& 4096 & $5.7 \mathrm{e}-06$ & 5 & $2.3 \mathrm{e}-05$ & $1.8 \mathrm{e}-05$ & 7 & $3.0 \mathrm{e}-05$ & $9.259 \mathrm{e}-03$ \\
\hline
\end{tabular}

Table 6: Absolute $\ell_{\infty}$ errors for methods listed from left to right: eigenfunction expansion with $m$ terms, Crank-Nicolson, rational Krylov projection with $p$ projection steps, and ode15s with average time step $\Delta t_{\text {avg }}$, for solving (2) to time $t_{f}$ on a $N$-point uniform grid with $u(x, 0)=\sin 2 x, \vec{\alpha}=[1,2,3,4]$ and $\vec{\rho}=[1 / 4,1 / 2,3 / 4]$.

\begin{tabular}{|l|c|c|c|c|c|c|c|c|}
\hline \multicolumn{2}{|c|}{} & \multicolumn{2}{c|}{ eig-exp } & C-N & \multicolumn{2}{c|}{ Krylov } & \multicolumn{2}{c|}{ ode15s } \\
\hline$t_{f}$ & $N$ & time & $m$ & time & time & $p$ & time & $\Delta t_{\text {avg }}$ \\
\hline 0.01 & 1024 & 0.019 & 29 & 0.134 & 0.055 & 17 & 0.049 & $1.852 \mathrm{e}-04$ \\
& 2048 & 0.020 & 29 & 0.329 & 0.082 & 17 & 0.097 & $1.852 \mathrm{e}-04$ \\
& 4096 & 0.033 & 29 & 0.623 & 0.135 & 17 & 0.147 & $1.786 \mathrm{e}-04$ \\
\hline 0.1 & 1024 & 0.007 & 11 & 0.142 & 0.025 & 11 & 0.056 & $1.266 \mathrm{e}-03$ \\
& 2048 & 0.008 & 11 & 0.362 & 0.069 & 11 & 0.135 & $1.250 \mathrm{e}-03$ \\
& 4096 & 0.012 & 11 & 0.526 & 0.070 & 11 & 0.210 & $1.220 \mathrm{e}-03$ \\
\hline 1 & 1024 & 0.003 & 3 & 0.193 & 0.021 & 7 & 0.087 & $9.709 \mathrm{e}-03$ \\
& 2048 & 0.003 & 3 & 0.288 & 0.028 & 7 & 0.144 & $9.615 \mathrm{e}-03$ \\
& 4096 & 0.005 & 3 & 0.642 & 0.054 & 7 & 0.285 & $9.434 \mathrm{e}-03$ \\
\hline
\end{tabular}

Table 7: Execution times (in seconds) for methods listed from left to right: eigenfunction expansion with $m$ terms, Crank-Nicolson, rational Krylov projection with $p$ projection steps, and ode15s with average time step $\Delta t_{\mathrm{avg}}$, for solving (2) to time $t_{f}$ on a $N$-point uniform grid with $u(x, 0)$ from $(37), \vec{\alpha}=[1,2,3,4]$ and $\vec{\rho}=[1 / 4,1 / 2,3 / 4]$.

\subsection{Self-adjoint Case}

\subsubsection{Computing Eigenvalues}

Tables 18-19 show the smallest ten nonzero eigenvalues of the operator $-\partial_{x} \alpha^{2}(x) \partial_{x}$, as computed using the $S A K$ principle with secant iteration, as in Section 3.2, and using the MatLab function eigs with a $N \times N$ matrix obtained via finite-element discretization. In Tables 18 and $20, N=2^{12}$, and in Tables 17 and 19, $N=2^{23}$. Tables 18 and 17 feature a coefficient with $n=4$ pieces, while $n=8$ in Tables 20 and 19. We observe similar accuracy and convergence behavior as in the non-self-adjoint case, with the exception of the smallest few eigenvalues. On the one hand, with these eigenvalues, we note that the reported error increases with $N$. On the other hand, as will be seen in Tables 29-32, in the case of final time $t_{f}=1$, the error in solutions of PDEs using these particular eigenvalues is quite small. This 


\begin{tabular}{|l|c|c|c|c|c|c|c|c|}
\hline \multicolumn{2}{|c|}{} & \multicolumn{2}{c|}{ eig-exp } & C-N & \multicolumn{2}{c|}{ Krylov } & \multicolumn{2}{c|}{ ode15s } \\
\hline$t_{f}$ & $N$ & error & $m$ & error & error & $p$ & error & $\Delta t_{\text {avg }}$ \\
\hline 0.01 & 1024 & $2.8 \mathrm{e}-03$ & 29 & $2.5 \mathrm{e}-04$ & $2.5 \mathrm{e}-04$ & 17 & $2.5 \mathrm{e}-04$ & $1.852 \mathrm{e}-04$ \\
& 2048 & $1.4 \mathrm{e}-03$ & 29 & $1.2 \mathrm{e}-04$ & $1.2 \mathrm{e}-04$ & 17 & $1.2 \mathrm{e}-04$ & $1.852 \mathrm{e}-04$ \\
& 4096 & $6.9 \mathrm{e}-04$ & 29 & $4.9 \mathrm{e}-05$ & $8.9 \mathrm{e}-05$ & 17 & $5.4 \mathrm{e}-05$ & $1.786 \mathrm{e}-04$ \\
\hline 0.1 & 1024 & $8.7 \mathrm{e}-04$ & 11 & $7.7 \mathrm{e}-04$ & $5.5 \mathrm{e}-04$ & 11 & $4.9 \mathrm{e}-04$ & $1.266 \mathrm{e}-03$ \\
& 2048 & $4.2 \mathrm{e}-04$ & 11 & $7.7 \mathrm{e}-04$ & $2.6 \mathrm{e}-04$ & 11 & $2.2 \mathrm{e}-04$ & $1.250 \mathrm{e}-03$ \\
& 4096 & $1.9 \mathrm{e}-04$ & 11 & $7.2 \mathrm{e}-04$ & $1.1 \mathrm{e}-04$ & 11 & $9.0 \mathrm{e}-05$ & $1.220 \mathrm{e}-03$ \\
\hline 1 & 1024 & $2.1 \mathrm{e}-04$ & 3 & $1.9 \mathrm{e}-02$ & $2.1 \mathrm{e}-04$ & 7 & $2.0 \mathrm{e}-04$ & $9.709 \mathrm{e}-03$ \\
& 2048 & $9.9 \mathrm{e}-05$ & 3 & $1.9 \mathrm{e}-02$ & $1.0 \mathrm{e}-04$ & 7 & $1.0 \mathrm{e}-04$ & $9.615 \mathrm{e}-03$ \\
& 4096 & $4.3 \mathrm{e}-05$ & 3 & $1.9 \mathrm{e}-02$ & $4.4 \mathrm{e}-05$ & 7 & $5.5 \mathrm{e}-05$ & $9.434 \mathrm{e}-03$ \\
\hline
\end{tabular}

Table 8: Absolute $\ell_{\infty}$ errors for methods listed from left to right: eigenfunction expansion with $m$ terms, Crank-Nicolson, rational Krylov projection with $p$ projection steps, and ode15s with average time step $\Delta t_{\mathrm{avg}}$, for solving (2) to time $t_{f}$ on a $N$-point uniform grid with $u(x, 0)$ from $(37), \vec{\alpha}=[1,2,3,4]$ and $\vec{\rho}=[1 / 4,1 / 2,3 / 4]$.

\begin{tabular}{|l|c|c|c|c|c|c|c|c|}
\hline \multicolumn{2}{|c|}{} & \multicolumn{2}{c|}{ eig-exp } & C-N & \multicolumn{2}{c|}{ Krylov } & \multicolumn{2}{c|}{ ode15s } \\
\hline$t_{f}$ & $N$ & time & $m$ & time & time & $p$ & time & $\Delta t_{\text {avg }}$ \\
\hline 0.01 & 1024 & 0.035 & 31 & 0.146 & 0.039 & 16 & 0.036 & $2.326 \mathrm{e}-04$ \\
& 2048 & 0.035 & 31 & 0.415 & 0.084 & 16 & 0.087 & $2.326 \mathrm{e}-04$ \\
& 4096 & 0.047 & 31 & 0.601 & 0.118 & 16 & 0.120 & $2.174 \mathrm{e}-04$ \\
\hline 0.1 & 1024 & 0.012 & 11 & 0.185 & 0.032 & 12 & 0.060 & $1.351 \mathrm{e}-03$ \\
& 2048 & 0.012 & 11 & 0.244 & 0.039 & 12 & 0.086 & $1.351 \mathrm{e}-03$ \\
& 4096 & 0.017 & 11 & 0.530 & 0.080 & 12 & 0.163 & $1.299 \mathrm{e}-03$ \\
\hline 1 & 1024 & 0.006 & 5 & 0.153 & 0.018 & 8 & 0.073 & $8.403 \mathrm{e}-03$ \\
& 2048 & 0.006 & 5 & 0.339 & 0.029 & 8 & 0.166 & $8.403 \mathrm{e}-03$ \\
& 4096 & 0.007 & 5 & 0.904 & 0.068 & 8 & 0.265 & $8.197 \mathrm{e}-03$ \\
\hline
\end{tabular}

Table 9: Execution times (in seconds) for methods listed from left to right: eigenfunction expansion with $m$ terms, Crank-Nicolson, rational Krylov projection with $p$ projection steps, and ode15s with average time step $\Delta t_{\text {avg }}$, for solving (2) to time $t_{f}$ on a $N$-point uniform grid with $u(x, 0)=\sin 2 x, \vec{\alpha}=[1,2,3,4,2,6,4]$ and $\vec{\rho}=[3 / 8,1 / 2,3 / 4,4 / 5,5 / 6,6 / 7]$.

suggests inaccuracy in the results obtained via eigs, which play no role in the computation of the reference solutions of PDEs.

\subsubsection{Solving PDEs}

Tables 21-32 compare the performance of the methods listed at the beginning of this section for solving the heat equation (3) at $t=t_{f}$ with the same choices of initial data and coefficient $\alpha(x)$ as in the non-self-adjoint case. We compute eigenvalues $\lambda_{0}, \lambda_{1}, \ldots, \lambda_{m}$ and eigenfunctions $V_{0}(x), V_{1}(x), \ldots, V_{m}(x)$ using the algorithm described in Section 3.2, where $m$ is chosen so that $e^{-\lambda_{j} t_{f}}<\epsilon$ for $j>m$ and $\epsilon=10^{-4}$. Then, the approximate solution is obtained from the eigenfunction expansion

$$
u(x, t) \approx \sum_{j=0}^{m} V_{j}(x) e^{-\lambda_{j} t} \frac{\left\langle V_{j}, u(\cdot, 0)\right\rangle}{\left\langle V_{j}, V_{j}\right\rangle}
$$




\begin{tabular}{|l|c|c|c|c|c|c|c|c|}
\hline \multicolumn{2}{|c|}{} & \multicolumn{2}{c|}{ eig-exp } & C-N & \multicolumn{2}{c|}{ Krylov } & \multicolumn{2}{c|}{ ode15s } \\
\hline$t_{f}$ & $N$ & error & $m$ & error & error & $p$ & error & $\Delta t_{\text {avg }}$ \\
\hline 0.01 & 1024 & $8.5 \mathrm{e}-03$ & 31 & $3.8 \mathrm{e}-03$ & $3.8 \mathrm{e}-03$ & 16 & $3.8 \mathrm{e}-03$ & $2.326 \mathrm{e}-04$ \\
& 2048 & $4.4 \mathrm{e}-03$ & 31 & $1.3 \mathrm{e}-03$ & $1.3 \mathrm{e}-03$ & 16 & $1.3 \mathrm{e}-03$ & $2.326 \mathrm{e}-04$ \\
& 4096 & $2.0 \mathrm{e}-03$ & 31 & $8.9 \mathrm{e}-04$ & $8.9 \mathrm{e}-04$ & 16 & $8.9 \mathrm{e}-04$ & $2.174 \mathrm{e}-04$ \\
\hline 0.1 & 1024 & $2.7 \mathrm{e}-03$ & 11 & $1.8 \mathrm{e}-03$ & $1.8 \mathrm{e}-03$ & 12 & $1.8 \mathrm{e}-03$ & $1.351 \mathrm{e}-03$ \\
& 2048 & $1.3 \mathrm{e}-03$ & 11 & $1.7 \mathrm{e}-03$ & $1.7 \mathrm{e}-03$ & 12 & $1.7 \mathrm{e}-03$ & $1.351 \mathrm{e}-03$ \\
& 4096 & $6.4 \mathrm{e}-04$ & 11 & $4.2 \mathrm{e}-04$ & $4.1 \mathrm{e}-04$ & 12 & $4.1 \mathrm{e}-04$ & $1.299 \mathrm{e}-03$ \\
\hline 1 & 1024 & $1.1 \mathrm{e}-03$ & 5 & $1.2 \mathrm{e}-03$ & $1.2 \mathrm{e}-03$ & 8 & $1.2 \mathrm{e}-03$ & $8.403 \mathrm{e}-03$ \\
& 2048 & $2.9 \mathrm{e}-04$ & 5 & $3.9 \mathrm{e}-04$ & $3.4 \mathrm{e}-04$ & 8 & $3.4 \mathrm{e}-04$ & $8.403 \mathrm{e}-03$ \\
& 4096 & $2.4 \mathrm{e}-04$ & 5 & $4.4 \mathrm{e}-04$ & $2.7 \mathrm{e}-04$ & 8 & $2.7 \mathrm{e}-04$ & $8.197 \mathrm{e}-03$ \\
\hline
\end{tabular}

Table 10: Absolute $\ell_{\infty}$ errors for methods listed from left to right: eigenfunction expansion with $m$ terms, Crank-Nicolson, rational Krylov projection with $p$ projection steps, and ode15s with average time step $\Delta t_{\mathrm{avg}}$, for solving (2) to time $t_{f}$ on a $N$-point uniform grid with $u(x, 0)=\sin 2 x, \vec{\alpha}=[1,2,3,4,2,6,4]$ and $\vec{\rho}=[3 / 8,1 / 2,3 / 4,4 / 5,5 / 6,6 / 7]$.

\begin{tabular}{|l|c|c|c|c|c|c|c|c|}
\hline \multicolumn{2}{|c|}{} & \multicolumn{2}{c|}{ eig-exp } & C-N & \multicolumn{2}{c|}{ Krylov } & \multicolumn{2}{c|}{ ode15s } \\
\hline$t_{f}$ & $N$ & time & $m$ & time & time & $p$ & time & $\Delta t_{\text {avg }}$ \\
\hline 0.01 & 1024 & 0.031 & 33 & 0.102 & 0.034 & 18 & 0.031 & $1.852 \mathrm{e}-04$ \\
& 2048 & 0.034 & 33 & 0.209 & 0.061 & 18 & 0.062 & $1.852 \mathrm{e}-04$ \\
& 4096 & 0.052 & 33 & 0.457 & 0.139 & 18 & 0.154 & $1.786 \mathrm{e}-04$ \\
\hline 0.1 & 1024 & 0.013 & 11 & 0.136 & 0.024 & 12 & 0.049 & $1.266 \mathrm{e}-03$ \\
& 2048 & 0.013 & 11 & 0.288 & 0.054 & 12 & 0.117 & $1.266 \mathrm{e}-03$ \\
& 4096 & 0.019 & 11 & 0.598 & 0.113 & 12 & 0.213 & $1.220 \mathrm{e}-03$ \\
\hline 1 & 1024 & 0.006 & 5 & 0.136 & 0.018 & 8 & 0.072 & $9.709 \mathrm{e}-03$ \\
& 2048 & 0.006 & 5 & 0.417 & 0.037 & 8 & 0.232 & $9.615 \mathrm{e}-03$ \\
& 4096 & 0.008 & 5 & 0.571 & 0.060 & 8 & 0.254 & $9.434 \mathrm{e}-03$ \\
\hline
\end{tabular}

Table 11: Execution times (in seconds) for methods listed from left to right: eigenfunction expansion with $m$ terms, Crank-Nicolson, rational Krylov projection with $p$ projection steps, and ode15s with average time step $\Delta t_{\text {avg }}$, for solving (2) to time $t_{f}$ on a $N$-point uniform grid with $u(x, 0)$ from (37), $\vec{\alpha}=[1,2,3,4,2,6,4]$ and $\vec{\rho}=[3 / 8,1 / 2,3 / 4,4 / 5,5 / 6,6 / 7]$.

where $\langle\cdot, \cdot\rangle$ is the standard inner product on $[0,2 \pi]$.

We observe some very similar patterns in the results as in the non-selfadjoint case. That is, our approach exhibits efficiency and scalability that is superior to that of Crank-Nicolson, Krylov projection or ode15s, while its accuracy again lags slightly behind that of the other methods for $t_{f}=0.01$ but catches up to and often surpasses the other methods as $t_{f}$ increases.

Substantially different behavior is observed regarding spatial accuracy. The seven-piece coefficient presents difficulty for all methods; convergence is first-order in space for our method, while sublinear convergence is shown by Krylov projection and ode15s and Crank-Nicolson does not show convergence at all. For the other choices of $\alpha(x)$, with four or eight pieces, our method and Krylov projection show second-order convergence in space, while convergence is slower or nonexistent for Crank-Nicolson and ode15s. 


\begin{tabular}{|l|c|c|c|c|c|c|c|c|}
\hline \multicolumn{2}{|c|}{} & \multicolumn{2}{c|}{ eig-exp } & C-N & \multicolumn{2}{c|}{ Krylov } & \multicolumn{2}{c|}{ ode15s } \\
\hline$t_{f}$ & $N$ & error & $m$ & error & error & $p$ & error & $\Delta t_{\text {avg }}$ \\
\hline 0.01 & 1024 & $4.1 \mathrm{e}-03$ & 33 & $9.9 \mathrm{e}-04$ & $9.9 \mathrm{e}-04$ & 18 & $9.9 \mathrm{e}-04$ & $1.852 \mathrm{e}-04$ \\
& 2048 & $2.3 \mathrm{e}-03$ & 33 & $4.9 \mathrm{e}-04$ & $5.0 \mathrm{e}-04$ & 18 & $4.8 \mathrm{e}-04$ & $1.852 \mathrm{e}-04$ \\
& 4096 & $9.5 \mathrm{e}-04$ & 33 & $2.2 \mathrm{e}-04$ & $2.3 \mathrm{e}-04$ & 18 & $2.1 \mathrm{e}-04$ & $1.786 \mathrm{e}-04$ \\
\hline 0.1 & 1024 & $1.8 \mathrm{e}-03$ & 11 & $1.0 \mathrm{e}-03$ & $1.0 \mathrm{e}-03$ & 12 & $9.7 \mathrm{e}-04$ & $1.266 \mathrm{e}-03$ \\
& 2048 & $1.1 \mathrm{e}-03$ & 11 & $1.0 \mathrm{e}-03$ & $5.0 \mathrm{e}-04$ & 12 & $4.6 \mathrm{e}-04$ & $1.266 \mathrm{e}-03$ \\
& 4096 & $3.8 \mathrm{e}-04$ & 11 & $7.3 \mathrm{e}-04$ & $1.9 \mathrm{e}-04$ & 12 & $1.7 \mathrm{e}-04$ & $1.220 \mathrm{e}-03$ \\
\hline 1 & 1024 & $5.9 \mathrm{e}-04$ & 5 & $1.9 \mathrm{e}-02$ & $5.9 \mathrm{e}-04$ & 8 & $5.7 \mathrm{e}-04$ & $9.709 \mathrm{e}-03$ \\
& 2048 & $3.1 \mathrm{e}-04$ & 5 & $1.9 \mathrm{e}-02$ & $3.0 \mathrm{e}-04$ & 8 & $2.8 \mathrm{e}-04$ & $9.615 \mathrm{e}-03$ \\
& 4096 & $1.2 \mathrm{e}-04$ & 5 & $1.9 \mathrm{e}-02$ & $1.2 \mathrm{e}-04$ & 8 & $1.0 \mathrm{e}-04$ & $9.434 \mathrm{e}-03$ \\
\hline
\end{tabular}

Table 12: Absolute $\ell_{\infty}$ errors for methods listed from left to right: eigenfunction expansion with $m$ terms, Crank-Nicolson, rational Krylov projection with $p$ projection steps, and ode15s with average time step $\Delta t_{\mathrm{avg}}$, for solving (2) to time $t_{f}$ on a $N$-point uniform grid with $u(x, 0)$ from $(37), \vec{\alpha}=[1,2,3,4,2,6,4]$ and $\vec{\rho}=[3 / 8,1 / 2,3 / 4,4 / 5,5 / 6,6 / 7]$.

\begin{tabular}{|l|c|c|c|c|c|c|c|c|}
\hline \multicolumn{2}{|c|}{} & \multicolumn{2}{c|}{ eig-exp } & C-N & \multicolumn{2}{c|}{ Krylov } & \multicolumn{2}{c|}{ ode15s } \\
\hline$t_{f}$ & $N$ & time & $m$ & time & time & $p$ & time & $\Delta t_{\text {avg }}$ \\
\hline 0.01 & 1024 & 0.020 & 21 & 0.112 & 0.027 & 13 & 0.034 & $1.887 \mathrm{e}-04$ \\
& 2048 & 0.022 & 21 & 0.218 & 0.043 & 13 & 0.081 & $1.818 \mathrm{e}-04$ \\
& 4096 & 0.027 & 19 & 0.446 & 0.082 & 13 & 0.119 & $1.754 \mathrm{e}-04$ \\
\hline 0.1 & 1024 & 0.008 & 7 & 0.119 & 0.015 & 8 & 0.055 & $1.176 \mathrm{e}-03$ \\
& 2048 & 0.008 & 7 & 0.435 & 0.034 & 8 & 0.113 & $1.163 \mathrm{e}-03$ \\
& 4096 & 0.009 & 7 & 0.531 & 0.054 & 8 & 0.171 & $1.124 \mathrm{e}-03$ \\
\hline 1 & 1024 & 0.004 & 3 & 0.104 & 0.009 & 6 & 0.054 & $9.259 \mathrm{e}-03$ \\
& 2048 & 0.004 & 3 & 0.260 & 0.022 & 6 & 0.106 & $9.174 \mathrm{e}-03$ \\
& 4096 & 0.006 & 3 & 0.621 & 0.044 & 6 & 0.227 & $8.929 \mathrm{e}-03$ \\
\hline
\end{tabular}

Table 13: Execution times (in seconds) for methods listed from left to right: eigenfunction expansion with $m$ terms, Crank-Nicolson, rational Krylov projection with $p$ projection steps, and ode15s with average time step $\Delta t_{\text {avg }}$, for solving (2) to time $t_{f}$ on a $N$-point uniform grid with $u(x, 0)=\sin 2 x, \vec{\alpha}=[1,5,3,4,2,7,6,8]$ and $\vec{\rho}=$ $[1 / 8,1 / 4,3 / 8,1 / 2,5 / 8,3 / 4,7 / 8]$.

We normally expect second-order accuracy from finite elements with piecewise linear basis functions. It makes sense that this accuracy is only observed in Krylov projection, and not in Crank-Nicolson or ode15s, due to the larger temporal error incurred by the latter two methods. The spatial accuracy of our approach is discussed further at the end of this section.

Figures 11-16 show the solutions computed by the different methods. As in the non-self-adjoint case, we see that, visually, the solutions match, except in the case of Crank-Nicolson with the smoothed characteristic function as initial data, where, in some cases, high-frequency oscillations can occur at the jumps in the initial data. 


\begin{tabular}{|l|c|c|c|c|c|c|c|c|}
\hline \multicolumn{2}{|c|}{} & \multicolumn{2}{c|}{ eig-exp } & C-N & \multicolumn{2}{c|}{ Krylov } & \multicolumn{2}{c|}{ ode15s } \\
\hline$t_{f}$ & $N$ & error & $m$ & error & error & $p$ & error & $\Delta t_{\text {avg }}$ \\
\hline 0.01 & 1024 & $1.4 \mathrm{e}-02$ & 21 & $3.5 \mathrm{e}-03$ & $3.6 \mathrm{e}-03$ & 13 & $3.5 \mathrm{e}-03$ & $1.887 \mathrm{e}-04$ \\
& 2048 & $6.7 \mathrm{e}-03$ & 21 & $1.7 \mathrm{e}-03$ & $1.7 \mathrm{e}-03$ & 13 & $1.7 \mathrm{e}-03$ & $1.818 \mathrm{e}-04$ \\
& 4096 & $3.2 \mathrm{e}-03$ & 19 & $7.1 \mathrm{e}-04$ & $7.2 \mathrm{e}-04$ & 13 & $7.1 \mathrm{e}-04$ & $1.754 \mathrm{e}-04$ \\
\hline 0.1 & 1024 & $3.9 \mathrm{e}-03$ & 7 & $3.4 \mathrm{e}-03$ & $3.4 \mathrm{e}-03$ & 8 & $3.4 \mathrm{e}-03$ & $1.176 \mathrm{e}-03$ \\
& 2048 & $1.8 \mathrm{e}-03$ & 7 & $1.6 \mathrm{e}-03$ & $1.6 \mathrm{e}-03$ & 8 & $1.6 \mathrm{e}-03$ & $1.163 \mathrm{e}-03$ \\
& 4096 & $8.3 \mathrm{e}-04$ & 7 & $6.8 \mathrm{e}-04$ & $6.9 \mathrm{e}-04$ & 8 & $6.7 \mathrm{e}-04$ & $1.124 \mathrm{e}-03$ \\
\hline 1 & 1024 & $2.7 \mathrm{e}-03$ & 3 & $3.1 \mathrm{e}-03$ & $2.7 \mathrm{e}-03$ & 6 & $2.7 \mathrm{e}-03$ & $9.259 \mathrm{e}-03$ \\
& 2048 & $1.3 \mathrm{e}-03$ & 3 & $1.6 \mathrm{e}-03$ & $1.3 \mathrm{e}-03$ & 6 & $1.3 \mathrm{e}-03$ & $9.174 \mathrm{e}-03$ \\
& 4096 & $5.4 \mathrm{e}-04$ & 3 & $8.9 \mathrm{e}-04$ & $5.5 \mathrm{e}-04$ & 6 & $5.6 \mathrm{e}-04$ & $8.929 \mathrm{e}-03$ \\
\hline
\end{tabular}

Table 14: Absolute $\ell_{\infty}$ errors for methods listed from left to right: eigenfunction expansion with $m$ terms, Crank-Nicolson, rational Krylov projection with $p$ projection steps, and ode15s with average time step $\Delta t_{\mathrm{avg}}$, for solving (2) to time $t_{f}$ on a $N$-point uniform grid with $u(x, 0)=\sin 2 x, \vec{\alpha}=[1,5,3,4,2,7,6,8]$ and $\vec{\rho}=[1 / 8,1 / 4,3 / 8,1 / 2,5 / 8,3 / 4,7 / 8]$.

\begin{tabular}{|l|c|c|c|c|c|c|c|c|}
\hline \multicolumn{2}{|c|}{} & \multicolumn{2}{c|}{ eig-exp } & C-N & \multicolumn{2}{c|}{ Krylov } & \multicolumn{2}{c|}{ ode15s } \\
\hline$t_{f}$ & $N$ & time & $m$ & time & time & $p$ & time & $\Delta t_{\text {avg }}$ \\
\hline 0.01 & 1024 & 0.020 & 21 & 0.123 & 0.048 & 15 & 0.044 & $1.351 \mathrm{e}-04$ \\
& 2048 & 0.025 & 21 & 0.281 & 0.059 & 15 & 0.109 & $1.333 \mathrm{e}-04$ \\
& 4096 & 0.032 & 21 & 0.622 & 0.117 & 15 & 0.186 & $1.316 \mathrm{e}-04$ \\
\hline 0.1 & 1024 & 0.008 & 7 & 0.123 & 0.020 & 9 & 0.056 & $1.010 \mathrm{e}-03$ \\
& 2048 & 0.009 & 7 & 0.235 & 0.030 & 9 & 0.116 & $1.010 \mathrm{e}-03$ \\
& 4096 & 0.011 & 7 & 0.477 & 0.057 & 9 & 0.257 & $1.000 \mathrm{e}-03$ \\
\hline 1 & 1024 & 0.004 & 3 & 0.108 & 0.012 & 6 & 0.063 & $8.403 \mathrm{e}-03$ \\
& 2048 & 0.004 & 3 & 0.251 & 0.019 & 6 & 0.146 & $8.333 \mathrm{e}-03$ \\
& 4096 & 0.006 & 3 & 0.461 & 0.039 & 6 & 0.232 & $8.264 \mathrm{e}-03$ \\
\hline
\end{tabular}

Table 15: Execution times (in seconds) for methods listed from left to right: eigenfunction expansion with $m$ terms, Crank-Nicolson, rational Krylov projection with $p$ projection steps, and ode15s with average time step $\Delta t_{\text {avg }}$, for solving (2) to time $t_{f}$ on a $N$-point uniform grid with $u(x, 0)$ from $(37), \vec{\alpha}=[1,5,3,4,2,7,6,8]$ and $\vec{\rho}=$ $[1 / 8,1 / 4,3 / 8,1 / 2,5 / 8,3 / 4,7 / 8]$.

\subsection{Discussion}

While the accuracy and efficiency of our method (for both self-adjoint and non-self-adjoint cases) do not compare favorably to those of the other methods featured in this section, our method is generally much faster, and accuracy does improve as $t_{f}$ approaches 1 . Regarding cases where our method may be slower, it is worth noting that this method is parallelizable, meaning each computation of an eigenfunction is independent of others. Therefore, future work allows this method to be easily improved upon for even greater speed.

In cases where our method is not sufficiently accurate, a different approach to numerical integration to obtain the coefficients in the eigenfunction expansion may be chosen. Currently, our method uses the composite trape- 


\begin{tabular}{|l|c|c|c|c|c|c|c|c|}
\hline \multicolumn{2}{|c|}{} & \multicolumn{2}{c|}{ eig-exp } & C-N & \multicolumn{2}{c|}{ Krylov } & \multicolumn{2}{c|}{ ode15s } \\
\hline$t_{f}$ & $N$ & error & $m$ & error & error & $p$ & error & $\Delta t_{\text {avg }}$ \\
\hline 0.01 & 1024 & $3.9 \mathrm{e}-03$ & 21 & $1.7 \mathrm{e}-03$ & $1.3 \mathrm{e}-03$ & 15 & $1.3 \mathrm{e}-03$ & $1.351 \mathrm{e}-04$ \\
& 2048 & $1.9 \mathrm{e}-03$ & 21 & $8.6 \mathrm{e}-04$ & $6.3 \mathrm{e}-04$ & 15 & $6.2 \mathrm{e}-04$ & $1.333 \mathrm{e}-04$ \\
& 4096 & $9.5 \mathrm{e}-04$ & 21 & $4.0 \mathrm{e}-04$ & $2.7 \mathrm{e}-04$ & 15 & $2.7 \mathrm{e}-04$ & $1.316 \mathrm{e}-04$ \\
\hline 0.1 & 1024 & $4.7 \mathrm{e}-04$ & 7 & $7.3 \mathrm{e}-03$ & $9.0 \mathrm{e}-04$ & 9 & $9.5 \mathrm{e}-04$ & $1.010 \mathrm{e}-03$ \\
& 2048 & $2.2 \mathrm{e}-04$ & 7 & $8.0 \mathrm{e}-03$ & $4.3 \mathrm{e}-04$ & 9 & $4.6 \mathrm{e}-04$ & $1.010 \mathrm{e}-03$ \\
& 4096 & $9.0 \mathrm{e}-05$ & 7 & $8.3 \mathrm{e}-03$ & $1.9 \mathrm{e}-04$ & 9 & $2.1 \mathrm{e}-04$ & $1.000 \mathrm{e}-03$ \\
\hline 1 & 1024 & $1.1 \mathrm{e}-04$ & 3 & $7.2 \mathrm{e}-02$ & $1.3 \mathrm{e}-04$ & 6 & $2.2 \mathrm{e}-04$ & $8.403 \mathrm{e}-03$ \\
& 2048 & $5.4 \mathrm{e}-05$ & 3 & $7.1 \mathrm{e}-02$ & $6.1 \mathrm{e}-05$ & 6 & $1.4 \mathrm{e}-04$ & $8.333 \mathrm{e}-03$ \\
& 4096 & $2.4 \mathrm{e}-05$ & 3 & $7.1 \mathrm{e}-02$ & $2.6 \mathrm{e}-05$ & 6 & $1.1 \mathrm{e}-04$ & $8.264 \mathrm{e}-03$ \\
\hline
\end{tabular}

Table 16: Absolute $\ell_{\infty}$ errors for methods listed from left to right: eigenfunction expansion with $m$ terms, Crank-Nicolson, rational Krylov projection with $p$ projection steps, and ode15s with average time step $\Delta t_{\mathrm{avg}}$, for solving (2) to time $t_{f}$ on a $N$-point uniform grid with $u(x, 0)$ from $(37), \vec{\alpha}=[1,5,3,4,2,7,6,8]$ and $\vec{\rho}=[1 / 8,1 / 4,3 / 8,1 / 2,5 / 8,3 / 4,7 / 8]$.
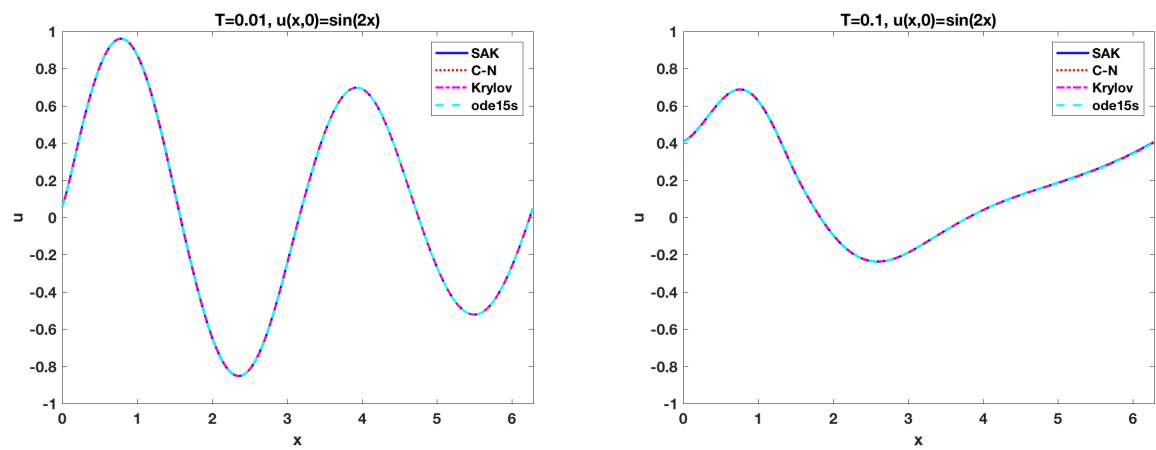

Figure 5: Solutions of $(2), u(x, 0)=\sin 2 x$, computed at $t_{f}=0.01,0.1$ on a uniform grid with $N=1,024$ points. The coefficient $\alpha^{2}(x)$ is defined by (1) with $\vec{\alpha}=[1,2,3,4]$, $\vec{\rho}=[1 / 4,1 / 2,3 / 4]$.

zoidal method of approximate integration, because of its known effectiveness with periodic functions, which applies to both the eigenfunctions and initial data. However, the error formula in the composite trapezoidal rule assumes a continuous second derivative, which the eigenfunctions do not have.

With smaller values of $t_{f}$, more terms are needed in the eigenfunction expansion, due to the slower decay of $e^{-\lambda_{j} t_{f}}$. For larger eigenvalues, the eigenfunctions are more oscillatory, thus making numerical integration more difficult. Future work for this method may involve applying Gaussian or adaptive quadrature methods corresponding to each piece of the piecewise constant coefficient instead, so as to improve accuracy. Therefore, while this method may not always perform as well as hoped based on the results presented in this paper, it can be effectively revised and enhanced in order 

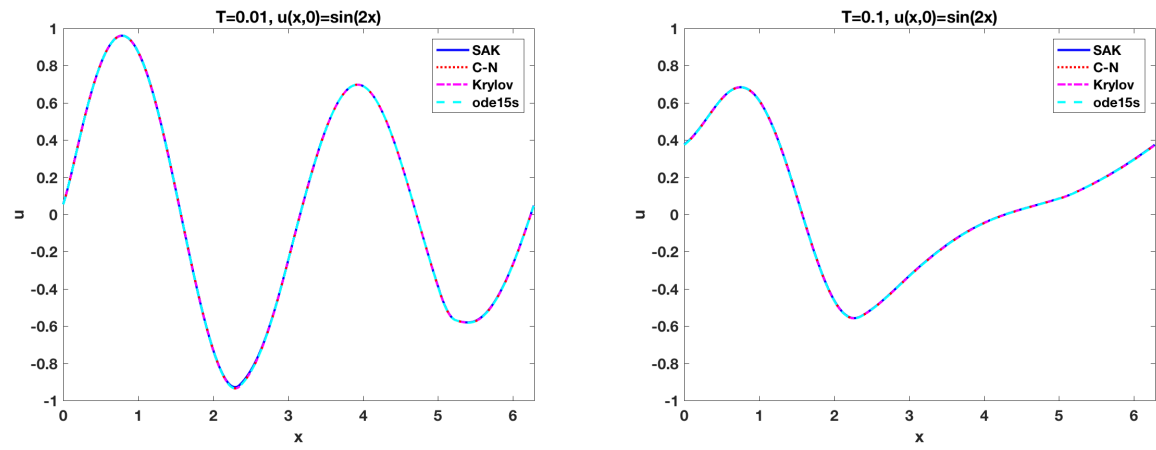

Figure 6: Solutions of $(2), u(x, 0)=\sin 2 x$, computed at $t_{f}=0.01,0.1$ on a uniform grid with $N=1,024$ points. The coefficient $\alpha^{2}(x)$ is defined by (1) with $\vec{\alpha}=[1,2,3,4,2,6,4]$, $\vec{\rho}=[3 / 8,1 / 2,3 / 4,4 / 5,5 / 6,6 / 7]$.
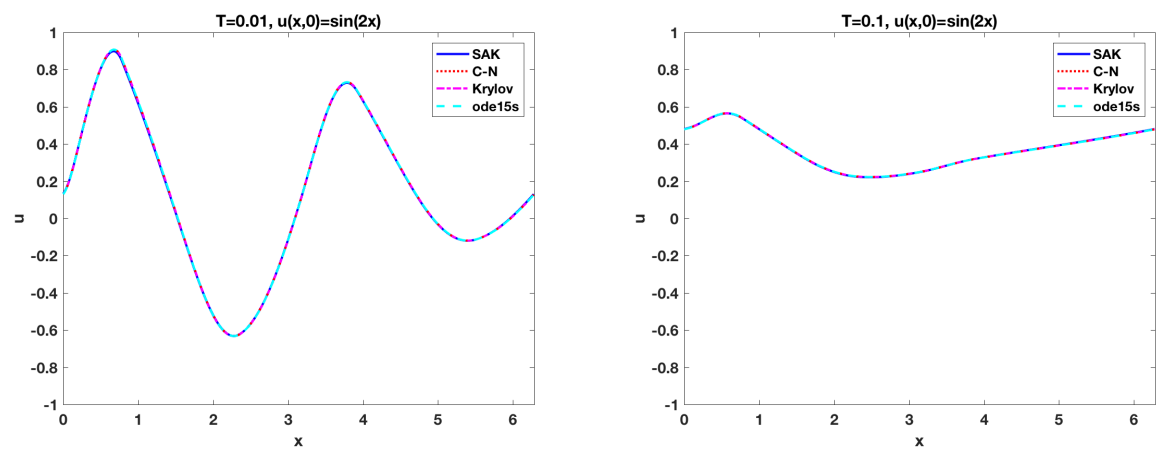

Figure 7: Solutions of $(2), u(x, 0)=\sin 2 x$, computed at $t_{f}=0.01,0.1$ on a uniform grid with $N=1,024$ points. The coefficient $\alpha^{2}(x)$ is defined by (1) with $\vec{\alpha}=[1,5,3,4,2,7,6,8]$, $\vec{\rho}=[1 / 8,1 / 4,3 / 8,1 / 2,5 / 8,3 / 4,7 / 8]$.

to deliver even faster and more accurate results than other methods.

\section{Conclusion}

We have developed an accurate, efficient, scalable, and parallelizable algorithm for computing eigenvalues and eigenfunctions of a second-order differential operator of the form $\alpha^{2}(x) \partial_{x x}$ or $\partial_{x} \alpha^{2}(x) \partial_{x}$, where $\alpha(x)$ is a piecewise constant coefficient with $n$ pieces, generalizing the work of [13] on the case $n=2$.

Here, we have used this eigensystem to solve diffusion equations, but it can also be used with other PDEs such as the wave equation by computing functions of eigenvalues such as $\cos \left(\sqrt{\lambda_{j}} t\right)$ rather than $e^{-\lambda_{j} t}$. We have also 

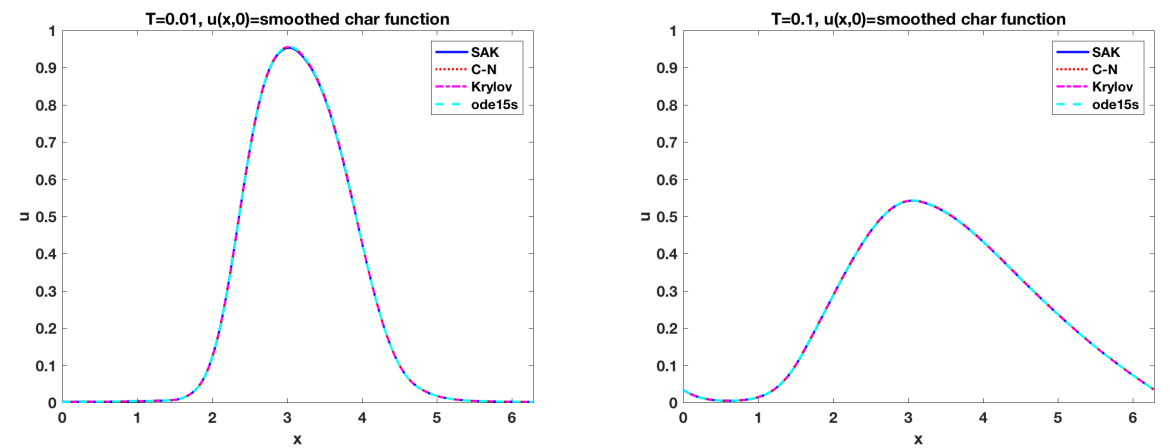

Figure 8: Solutions of $(2), u(x, 0)$ from (37), computed at $t_{f}=0.01,0.1$ on a uniform grid with $N=1,024$ points. The coefficient $\alpha^{2}(x)$ is defined by (1) with $\vec{\alpha}=[1,2,3,4]$, $\vec{\rho}=[1 / 4,1 / 2,3 / 4]$.
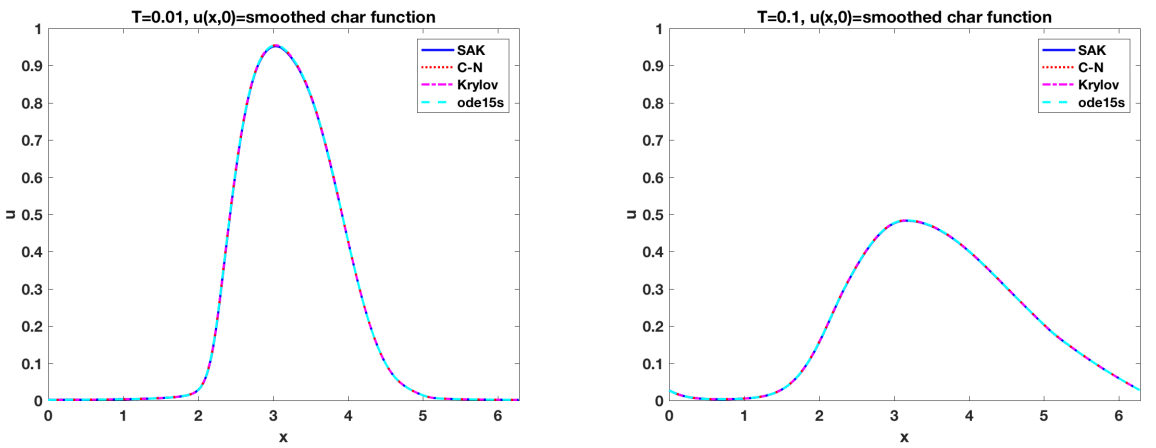

Figure 9: Solutions of $(2), u(x, 0)$ from (37), computed at $t_{f}=0.01,0.1$ on a uniform grid with $N=1,024$ points. The coefficient $\alpha^{2}(x)$ is defined by (1) with $\vec{\alpha}=[1,2,3,4,2,6,4]$, $\vec{\rho}=[3 / 8,1 / 2,3 / 4,4 / 5,5 / 6,6 / 7]$.

used periodic boundary conditions, but the same idea can be applied with homogeneous Dirichlet or Neumann boundary conditions by replacing the periodicity conditions. The resulting matrix $M$ still has the same structure.

Ongoing work considers generalization to higher spatial dimensions, as the $S A K$ principle is not limited to $1-\mathrm{D}$ operators. This generalization is complicated by the need to include eigenfunctions of other forms, involving hyperbolic functions [22]. It is also of interest to consider the limit as $n \rightarrow$ $\infty$, in which case approximate eigenfunctions for operators with smoothly varying coefficients could be obtained.

Another future direction of interest would be the case in which the PDE includes an advection term, with a coefficient that is also piecewise constant. 

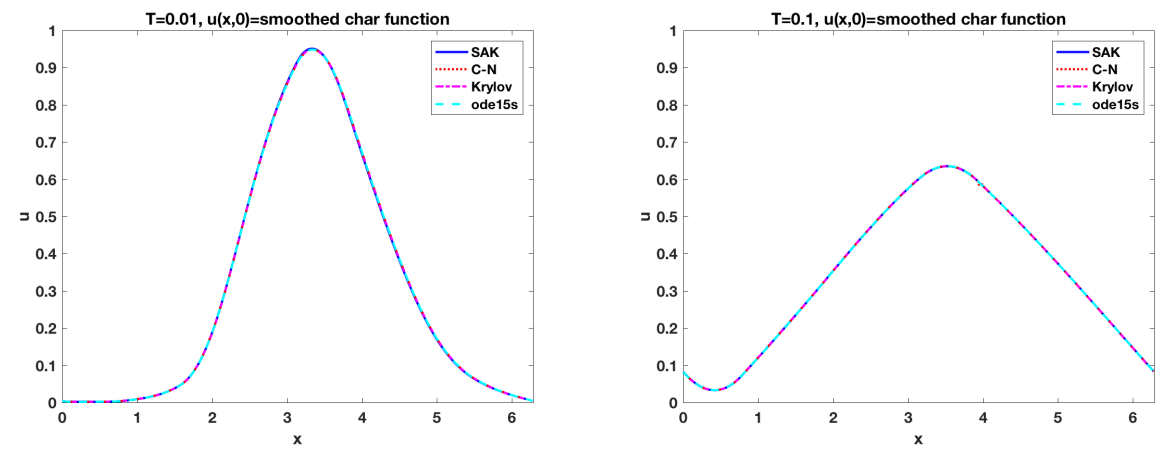

Figure 10: Solutions of (2), $u(x, 0)$ from (37), computed at $t_{f}=0.01,0.1$ on a uniform grid with $N=1,024$ points. The coefficient $\alpha^{2}(x)$ is defined by (1) with $\vec{\alpha}=[1,5,3,4,2,7,6,8]$, $\vec{\rho}=[1 / 8,1 / 4,3 / 8,1 / 2,5 / 8,3 / 4,7 / 8]$.

\begin{tabular}{rrrrr}
\hline$j$ & $\lambda_{j}(S A K)$ & $\lambda_{j}(\mathrm{eig})$ & iterations & relative err \\
\hline 1 & 3.097941505033577 & 3.097940432538863 & 5 & $3.46 \mathrm{e}-07$ \\
2 & 4.251422728928484 & 4.251420921975639 & 6 & $4.25 \mathrm{e}-07$ \\
3 & 13.537755713873489 & 13.537739478497940 & 5 & $1.20 \mathrm{e}-06$ \\
4 & 15.668508094554506 & 15.668473921612334 & 6 & $2.18 \mathrm{e}-06$ \\
5 & 31.902087233265412 & 31.901978395077720 & 5 & $3.41 \mathrm{e}-06$ \\
6 & 35.425319948808259 & 35.425181211006119 & 7 & $3.92 \mathrm{e}-06$ \\
7 & 56.411139858260810 & 56.410868559504088 & 6 & $4.81 \mathrm{e}-06$ \\
8 & 61.429391470973243 & 61.428874641328655 & 6 & $8.41 \mathrm{e}-06$ \\
9 & 89.272086669374303 & 89.271357580715517 & 6 & $8.17 \mathrm{e}-06$ \\
10 & 95.183229810726530 & 95.182094519308265 & 6 & $1.19 \mathrm{e}-05$ \\
\hline
\end{tabular}

Table 17: Eigenvalues computed using the $S A K$ principle with secant iteration, and eigs on a finite-difference matrix, of the operator $-\alpha^{2}(x) \partial_{x}^{2}$ with $\vec{\alpha}=[1,2,3,4], \vec{\rho}=$ $[1 / 4,1 / 2,3 / 4]$ and $N=4,096$ grid points.

The case of a piecewise constant advection coefficient, but with a constant diffusion coefficient, was considered in [23]. However, this is a particularly challenging problem, as the approach used in this paper cannot be modified in a straightforward manner to handle this case. In particular, it has been observed that the eigenfunctions do not have a constant amplitude on each piece.

\section{Acknowledgment}

The first author was partially supported by a grant from the Center for Undergraduate Research at The University of Southern Mississippi.

[1] G. B. Folland, Introduction to Partial Differential Equations, 2nd Edition, Princeton University Press, 1995. 


\begin{tabular}{rrrrr}
\hline$j$ & $\lambda_{j}(S A K)$ & $\lambda_{j}(\mathrm{eig})$ & iterations & relative err \\
\hline 1 & 3.097941505033577 & 3.097926927925785 & 5 & $4.71 \mathrm{e}-06$ \\
2 & 4.251422728928484 & 4.251418333598331 & 6 & $1.03 \mathrm{e}-06$ \\
3 & 13.537755713873489 & 13.537737194958565 & 5 & $1.37 \mathrm{e}-06$ \\
4 & 15.668508094554506 & 15.668507008875586 & 6 & $6.93 \mathrm{e}-08$ \\
5 & 31.902087233265412 & 31.902078078045157 & 5 & $2.87 \mathrm{e}-07$ \\
6 & 35.425319948808259 & 35.425313532758977 & 7 & $1.81 \mathrm{e}-07$ \\
7 & 56.411139858260810 & 56.411119659042910 & 6 & $3.58 \mathrm{e}-07$ \\
8 & 61.429391470973243 & 61.429390833928522 & 6 & $1.04 \mathrm{e}-08$ \\
9 & 89.272086669374303 & 89.272071303807280 & 6 & $1.72 \mathrm{e}-07$ \\
10 & 95.183229810726530 & 95.183228514110098 & 6 & $1.36 \mathrm{e}-08$ \\
\hline
\end{tabular}

Table 18: Eigenvalues computed using the $S A K$ principle with secant iteration, and eigs on a finite-difference matrix, of the operator $-\partial_{x} \alpha^{2}(x) \partial_{x}$ with $\vec{\alpha}=[1,2,3,4]$, $\vec{\rho}=[1 / 4,1 / 2,3 / 4]$ and $N=8,388,608$ grid points.

\begin{tabular}{rrrrr}
\hline$j$ & $\lambda_{j}(S A K)$ & $\lambda_{j}(\mathrm{eig})$ & iterations & relative err \\
\hline 1 & 3.9679747239207 & 3.9679723630579 & 5 & $5.95 \mathrm{e}-07$ \\
2 & 12.6253580194816 & 12.6253454562482 & 5 & $9.95 \mathrm{e}-07$ \\
3 & 24.2171296638348 & 24.2170508527271 & 7 & $3.25 \mathrm{e}-06$ \\
4 & 46.0526204562029 & 46.0525391780218 & 5 & $1.76 \mathrm{e}-06$ \\
5 & 68.5575768148243 & 68.5568461105957 & 5 & $1.07 \mathrm{e}-05$ \\
6 & 82.7275880706043 & 82.7273505060318 & 6 & $2.87 \mathrm{e}-06$ \\
7 & 121.7888914858721 & 121.7881800573054 & 5 & $5.84 \mathrm{e}-06$ \\
8 & 145.2960020397981 & 145.2925406014834 & 7 & $2.38 \mathrm{e}-05$ \\
9 & 209.7194743032816 & 209.7176162644602 & 6 & $8.86 \mathrm{e}-06$ \\
10 & 242.6942814147148 & 242.6866432428965 & 7 & $3.15 \mathrm{e}-05$
\end{tabular}

Table 19: Eigenvalues computed using the $S A K$ principle with secant iteration, and eigs on a finite-difference matrix, of the operator $-\partial_{x} \alpha^{2}(x) \partial_{x}$ with $\vec{\alpha}=[1,5,3,4,2,7,6,8]$, $\vec{\rho}=[1 / 8,1 / 4,3 / 8,1 / 2,5 / 8,3 / 4,7 / 8]$ and $N=4,096$ grid points.

[2] N. I. Portenko, Generalized diffusion processes, Vol. 83 of Translations of Mathematical Monographs, American Mathematical Society, 1990.

[3] P. Étoré, On random walk simulation of one-dimensional diffusion processes with discontinuous coefficients, Electron. J. Probab. 11 (9) (2006) 249-275.

[4] H. Hoteit, R. Mose, A. Younes, F. Lehmann, P. Ackerer, Threedimensional modeling of mass transfer in porous media using the mixed hybrid finite elements and the random-walk methods, Mathematical Geology 34 (4) (2002) 435-456.

[5] E. M. LaBolle, G. E. Fogg, A. F. B. Tompson, Random-walk simulation of transport in heterogeneous porous media: Local mass-conservation problem and implementation methods, Water Resources Research 32 (3) (1996) 583-593. 


\begin{tabular}{rrrrr}
\hline$j$ & $\lambda_{j}(S A K)$ & $\lambda_{j}(\mathrm{eig})$ & iterations & relative err \\
\hline 1 & 3.9679747239207 & 3.9679069688242 & 5 & $1.71 \mathrm{e}-05$ \\
2 & 12.6253580194816 & 12.6253598638896 & 5 & $1.46 \mathrm{e}-07$ \\
3 & 24.2171296638348 & 24.2171398039054 & 7 & $4.19 \mathrm{e}-07$ \\
4 & 46.0526204562029 & 46.0525491383453 & 5 & $1.55 \mathrm{e}-06$ \\
5 & 68.5575768148243 & 68.5575675147394 & 5 & $1.36 \mathrm{e}-07$ \\
6 & 82.7275880706043 & 82.7274934271921 & 6 & $1.14 \mathrm{e}-06$ \\
7 & 121.7888914858721 & 121.7888797739920 & 5 & $9.62 \mathrm{e}-08$ \\
8 & 145.2960020397981 & 145.2959943106795 & 7 & $5.32 \mathrm{e}-08$ \\
9 & 209.7194743032816 & 209.7194536976682 & 6 & $9.83 \mathrm{e}-08$ \\
10 & 242.6942814147148 & 242.6942602525224 & 7 & $8.72 \mathrm{e}-08$ \\
\hline
\end{tabular}

Table 20: Eigenvalues computed using the $S A K$ principle with secant iteration, and eigs on a finite-difference matrix, of the operator $-\partial_{x} \alpha^{2}(x) \partial_{x}$ with $\vec{\alpha}=[1,5,3,4,2,7,6,8]$, $\vec{\rho}=[1 / 8,1 / 4,3 / 8,1 / 2,5 / 8,3 / 4,7 / 8]$ and $N=8,388,608$ grid points.

\begin{tabular}{|l|c|c|c|c|c|c|c|c|}
\hline \multicolumn{2}{|c|}{} & \multicolumn{2}{c|}{ eig-exp } & C-N & \multicolumn{2}{c|}{ Krylov } & \multicolumn{2}{c|}{ ode15s } \\
\hline$t_{f}$ & $N$ & time & $m$ & time & time & $p$ & time & $\Delta t_{\text {avg }}$ \\
\hline 0.01 & 1024 & 0.033 & 39 & 0.061 & 0.070 & 26 & 0.074 & $1.064 \mathrm{e}-04$ \\
& 2048 & 0.033 & 39 & 0.113 & 0.238 & 26 & 0.300 & $9.091 \mathrm{e}-05$ \\
& 4096 & 0.048 & 39 & 0.187 & 0.118 & 26 & 0.298 & $8.130 \mathrm{e}-05$ \\
\hline 0.1 & 1024 & 0.011 & 13 & 0.043 & 0.019 & 15 & 0.074 & $7.519 \mathrm{e}-04$ \\
& 2048 & 0.013 & 13 & 0.084 & 0.033 & 15 & 0.169 & $6.623 \mathrm{e}-04$ \\
& 4096 & 0.016 & 13 & 0.184 & 0.063 & 15 & 0.723 & $5.988 \mathrm{e}-04$ \\
\hline 1 & 1024 & 0.005 & 5 & 0.045 & 0.010 & 9 & 0.091 & $5.495 \mathrm{e}-03$ \\
& 2048 & 0.006 & 5 & 0.078 & 0.016 & 9 & 0.206 & $4.975 \mathrm{e}-03$ \\
& 4096 & 0.007 & 5 & 0.167 & 0.039 & 9 & 0.400 & $4.545 \mathrm{e}-03$ \\
\hline
\end{tabular}

Table 21: Execution times (in seconds) for methods listed from left to right: eigenfunction expansion with $m$ terms, Crank-Nicolson, rational Krylov projection with $p$ projection steps, and ode15s with average time step $\Delta t_{\text {avg }}$, for solving (3) to time $t_{f}$ on a $N$-point uniform grid with $u(x, 0)=\sin 2 x, \vec{\alpha}=[1,2,3,4]$ and $\vec{\rho}=[1 / 4,1 / 2,3 / 4]$.

[6] A. Lejay, G. Pichot, Simulating diffusion processes in discontinuous media: A numerical scheme with constant time steps, Journal of Computational Physics 231 (21) (2012) 7299-7314.

[7] G. J. M. Uffink, A random walk method for the simulation of macrodispersion in a stratified aquifer, Relation of Groundwater Quantity and Quality 146 (1985) 103-114.

[8] B. Gaveau, M. Okada, T. Okada, Second order differential operators and dirichlet integrals with singular coefficients, Tohoku Math. Journ. 39 (1987) 465-504.

[9] A. Lejay, L. Lenôtre, G. Pichot, Analytic expressions of the solutions of advection-diffusion problems in $1 \mathrm{~d}$ with discontinuous coefficients, preprint (2017). 


\begin{tabular}{|l|c|c|c|c|c|c|c|c|}
\hline \multicolumn{2}{|c|}{} & \multicolumn{2}{c|}{ eig-exp } & C-N & \multicolumn{2}{c|}{ Krylov } & \multicolumn{2}{c|}{ ode15s } \\
\hline$t_{f}$ & $N$ & error & $m$ & error & error & $p$ & error & $\Delta t_{\text {avg }}$ \\
\hline 0.01 & 1024 & $5.6 \mathrm{e}-06$ & 39 & $4.3 \mathrm{e}-05$ & $2.7 \mathrm{e}-05$ & 26 & $3.2 \mathrm{e}-05$ & $1.064 \mathrm{e}-04$ \\
& 2048 & $1.4 \mathrm{e}-06$ & 39 & $4.0 \mathrm{e}-04$ & $6.7 \mathrm{e}-06$ & 26 & $9.6 \mathrm{e}-06$ & $9.091 \mathrm{e}-05$ \\
& 4096 & $3.4 \mathrm{e}-07$ & 39 & $1.0 \mathrm{e}-03$ & $1.5 \mathrm{e}-06$ & 26 & $6.7 \mathrm{e}-06$ & $8.130 \mathrm{e}-05$ \\
\hline 0.1 & 1024 & $5.2 \mathrm{e}-07$ & 13 & $2.6 \mathrm{e}-03$ & $5.4 \mathrm{e}-06$ & 15 & $5.0 \mathrm{e}-06$ & $7.519 \mathrm{e}-04$ \\
& 2048 & $1.4 \mathrm{e}-07$ & 13 & $4.3 \mathrm{e}-03$ & $1.4 \mathrm{e}-06$ & 15 & $1.5 \mathrm{e}-06$ & $6.623 \mathrm{e}-04$ \\
& 4096 & $4.6 \mathrm{e}-08$ & 13 & $5.1 \mathrm{e}-03$ & $4.1 \mathrm{e}-07$ & 15 & $2.1 \mathrm{e}-06$ & $5.988 \mathrm{e}-04$ \\
\hline 1 & 1024 & $6.8 \mathrm{e}-09$ & 5 & $1.5 \mathrm{e}-02$ & $6.8 \mathrm{e}-07$ & 9 & $1.1 \mathrm{e}-06$ & $5.495 \mathrm{e}-03$ \\
& 2048 & $3.1 \mathrm{e}-09$ & 5 & $1.7 \mathrm{e}-02$ & $1.7 \mathrm{e}-07$ & 9 & $4.7 \mathrm{e}-07$ & $4.975 \mathrm{e}-03$ \\
& 4096 & $2.7 \mathrm{e}-09$ & 5 & $1.7 \mathrm{e}-02$ & $3.8 \mathrm{e}-08$ & 9 & $1.4 \mathrm{e}-06$ & $4.545 \mathrm{e}-03$ \\
\hline
\end{tabular}

Table 22: Absolute $\ell_{\infty}$ errors for methods listed from left to right: eigenfunction expansion with $m$ terms, Crank-Nicolson, rational Krylov projection with $p$ projection steps, and ode15s with average time step $\Delta t_{\text {avg }}$, for solving (3) to time $t_{f}$ on a $N$-point uniform grid with $u(x, 0)=\sin 2 x, \vec{\alpha}=[1,2,3,4]$ and $\vec{\rho}=[1 / 4,1 / 2,3 / 4]$.

\begin{tabular}{|l|c|c|c|c|c|c|c|c|}
\hline \multicolumn{2}{|c|}{} & \multicolumn{2}{c|}{ eig-exp } & C-N & \multicolumn{2}{c|}{ Krylov } & \multicolumn{2}{c|}{ ode15s } \\
\hline$t_{f}$ & $N$ & time & $m$ & time & time & $p$ & time & $\Delta t_{\text {avg }}$ \\
\hline 0.01 & 1024 & 0.031 & 37 & 0.052 & 0.046 & 26 & 0.042 & $1.786 \mathrm{e}-04$ \\
& 2048 & 0.034 & 37 & 0.086 & 0.059 & 26 & 0.074 & $1.754 \mathrm{e}-04$ \\
& 4096 & 0.049 & 37 & 0.153 & 0.096 & 26 & 0.144 & $1.695 \mathrm{e}-04$ \\
\hline 0.1 & 1024 & 0.012 & 13 & 0.046 & 0.018 & 15 & 0.057 & $1.220 \mathrm{e}-03$ \\
& 2048 & 0.012 & 13 & 0.097 & 0.029 & 15 & 0.096 & $1.205 \mathrm{e}-03$ \\
& 4096 & 0.016 & 13 & 0.175 & 0.058 & 15 & 0.227 & $1.176 \mathrm{e}-03$ \\
\hline 1 & 1024 & 0.005 & 5 & 0.039 & 0.011 & 9 & 0.058 & $9.434 \mathrm{e}-03$ \\
& 2048 & 0.006 & 5 & 0.081 & 0.018 & 9 & 0.121 & $9.259 \mathrm{e}-03$ \\
& 4096 & 0.009 & 5 & 0.236 & 0.038 & 9 & 0.359 & $9.091 \mathrm{e}-03$ \\
\hline
\end{tabular}

Table 23: Execution times (in seconds) for methods listed from left to right: eigenfunction expansion with $m$ terms, Crank-Nicolson, rational Krylov projection with $p$ projection steps, and ode15s with average time step $\Delta t_{\mathrm{avg}}$, for solving (3) to time $t_{f}$ on a $N$-point uniform grid with $u(x, 0)$ from $(37), \vec{\alpha}=[1,2,3,4]$ and $\vec{\rho}=[1 / 4,1 / 2,3 / 4]$.

[10] L. Gerardo-Giorda, P. Le Tallec, F. Nataf, A robin-robin preconditioner for advection-diffusion equations with discontinuous coefficients, Computer Methods in Applied Mechanics and Engineering 193 (9-11) (2004) $745-764$.

[11] P. Zunino, Iterative substructuring methods for advection-diffusion problems in heterogeneous media, in: E. Bänsch (Ed.), Challenges in Scientific Computing - CISC 2002, Vol. 35 of Lecture Notes in Computational Science and Engineering, Springer, 2003, pp. 184-210.

[12] B. Gustafsson, H. O. Kreiss, J. Oliger, Time Dependent Problems And Difference Methods, John Wiley \& Sons, Inc., 1995.

[13] E. M. Garon, J. V. Lambers, Modeling the diffusion of heat energy 


\begin{tabular}{|l|c|c|c|c|c|c|c|c|}
\hline \multicolumn{2}{|c|}{} & \multicolumn{2}{c|}{ eig-exp } & C-N & \multicolumn{2}{c|}{ Krylov } & \multicolumn{2}{c|}{ ode15s } \\
\hline$t_{f}$ & $N$ & error & $m$ & error & error & $p$ & error & $\Delta t_{\text {avg }}$ \\
\hline 0.01 & 1024 & $6.2 \mathrm{e}-06$ & 37 & $7.3 \mathrm{e}-06$ & $9.2 \mathrm{e}-06$ & 26 & $2.2 \mathrm{e}-05$ & $1.786 \mathrm{e}-04$ \\
& 2048 & $1.5 \mathrm{e}-06$ & 37 & $1.2 \mathrm{e}-06$ & $2.3 \mathrm{e}-06$ & 26 & $2.0 \mathrm{e}-05$ & $1.754 \mathrm{e}-04$ \\
& 4096 & $3.8 \mathrm{e}-07$ & 37 & $1.9 \mathrm{e}-06$ & $6.2 \mathrm{e}-07$ & 26 & $1.4 \mathrm{e}-05$ & $1.695 \mathrm{e}-04$ \\
\hline 0.1 & 1024 & $5.5 \mathrm{e}-07$ & 13 & $5.3 \mathrm{e}-04$ & $3.2 \mathrm{e}-06$ & 15 & $2.5 \mathrm{e}-05$ & $1.220 \mathrm{e}-03$ \\
& 2048 & $1.4 \mathrm{e}-07$ & 13 & $6.6 \mathrm{e}-04$ & $7.7 \mathrm{e}-07$ & 15 & $2.8 \mathrm{e}-05$ & $1.205 \mathrm{e}-03$ \\
& 4096 & $3.6 \mathrm{e}-08$ & 13 & $6.8 \mathrm{e}-04$ & $2.0 \mathrm{e}-07$ & 15 & $2.8 \mathrm{e}-05$ & $1.176 \mathrm{e}-03$ \\
\hline 1 & 1024 & $2.9 \mathrm{e}-08$ & 5 & $1.9 \mathrm{e}-02$ & $2.6 \mathrm{e}-07$ & 9 & $8.6 \mathrm{e}-05$ & $9.434 \mathrm{e}-03$ \\
& 2048 & $7.1 \mathrm{e}-09$ & 5 & $1.9 \mathrm{e}-02$ & $6.5 \mathrm{e}-08$ & 9 & $9.2 \mathrm{e}-05$ & $9.259 \mathrm{e}-03$ \\
& 4096 & $1.8 \mathrm{e}-09$ & 5 & $1.9 \mathrm{e}-02$ & $1.7 \mathrm{e}-08$ & 9 & $6.8 \mathrm{e}-05$ & $9.091 \mathrm{e}-03$ \\
\hline
\end{tabular}

Table 24: Absolute $\ell_{\infty}$ errors for methods listed from left to right: eigenfunction expansion with $m$ terms, Crank-Nicolson, rational Krylov projection with $p$ projection steps, and ode15s with average time step $\Delta t_{\mathrm{avg}}$, for solving (3) to time $t_{f}$ on a $N$-point uniform grid with $u(x, 0)$ from $(37), \vec{\alpha}=[1,2,3,4]$ and $\vec{\rho}=[1 / 4,1 / 2,3 / 4]$.

\begin{tabular}{|l|c|c|c|c|c|c|c|c|}
\hline \multicolumn{2}{|c|}{} & \multicolumn{2}{c|}{ eig-exp } & C-N & \multicolumn{2}{c|}{ Krylov } & \multicolumn{2}{c|}{ ode15s } \\
\hline$t_{f}$ & $N$ & time & $m$ & time & time & $p$ & time & $\Delta t_{\text {avg }}$ \\
\hline 0.01 & 1024 & 0.081 & 43 & 0.054 & 0.041 & 25 & 0.086 & $1.031 \mathrm{e}-04$ \\
& 2048 & 0.079 & 43 & 0.109 & 0.092 & 25 & 0.179 & $8.929 \mathrm{e}-05$ \\
& 4096 & 0.105 & 43 & 0.266 & 0.166 & 25 & 0.510 & $7.874 \mathrm{e}-05$ \\
\hline 0.1 & 1024 & 0.042 & 15 & 0.062 & 0.032 & 16 & 0.125 & $7.519 \mathrm{e}-04$ \\
& 2048 & 0.045 & 15 & 0.162 & 0.071 & 16 & 0.299 & $6.623 \mathrm{e}-04$ \\
& 4096 & 0.043 & 15 & 0.630 & 0.225 & 16 & 1.952 & $6.061 \mathrm{e}-04$ \\
\hline 1 & 1024 & 0.011 & 5 & 0.093 & 0.027 & 9 & 0.266 & $5.495 \mathrm{e}-03$ \\
& 2048 & 0.011 & 5 & 0.207 & 0.053 & 9 & 0.700 & $5.000 \mathrm{e}-03$ \\
& 4096 & 0.012 & 5 & 0.360 & 0.090 & 9 & 2.429 & $4.630 \mathrm{e}-03$ \\
\hline
\end{tabular}

Table 25: Execution times (in seconds) for methods listed from left to right: eigenfunction expansion with $m$ terms, Crank-Nicolson, rational Krylov projection with $p$ projection steps, and ode15s with average time step $\Delta t_{\text {avg }}$, for solving (3) to time $t_{f}$ on a $N$-point uniform grid with $u(x, 0)=\sin 2 x, \vec{\alpha}=[1,2,3,4,2,6,4]$ and $\vec{\rho}=[3 / 8,1 / 2,3 / 4,4 / 5,5 / 6,6 / 7]$.

within composites of homogeneous materials using the uncertainty principle, Computational and Applied Mathematics (2017) In press.

[14] M. Filoche, S. Mayaboroda, B. Patterson, Localization of eigenfunctions of a one-dimensional elliptic operator, Contemporary Mathematics 581 (2012) 99-116.

[15] C. Fefferman, The uncertainty principle, Bulletin of the American Mathematical Society 9 (1983) 129-206.

[16] W. Heisenberg, Über den anschaulichen inhalt der quantentheoretischen kinematik und mechanik, Zeitschrift für Physik 43 (3-4) (1927) 172-198.

[17] W. O. Amrein, A. M. Berthier, On support properties of lp-functions 


\begin{tabular}{|l|c|c|c|c|c|c|c|c|}
\hline \multicolumn{2}{|c|}{} & \multicolumn{2}{c|}{ eig-exp } & C-N & \multicolumn{2}{c|}{ Krylov } & \multicolumn{2}{c|}{ ode15s } \\
\hline$t_{f}$ & $N$ & error & $m$ & error & error & $p$ & error & $\Delta t_{\text {avg }}$ \\
\hline 0.01 & 1024 & $1.0 \mathrm{e}-02$ & 43 & $2.1 \mathrm{e}-03$ & $2.1 \mathrm{e}-03$ & 25 & $2.1 \mathrm{e}-03$ & $1.031 \mathrm{e}-04$ \\
& 2048 & $1.0 \mathrm{e}-02$ & 43 & $1.3 \mathrm{e}-03$ & $1.1 \mathrm{e}-03$ & 25 & $1.1 \mathrm{e}-03$ & $8.929 \mathrm{e}-05$ \\
& 4096 & $1.0 \mathrm{e}-02$ & 43 & $1.1 \mathrm{e}-03$ & $3.8 \mathrm{e}-04$ & 25 & $3.8 \mathrm{e}-04$ & $7.874 \mathrm{e}-05$ \\
\hline 0.1 & 1024 & $4.9 \mathrm{e}-04$ & 15 & $2.8 \mathrm{e}-03$ & $4.5 \mathrm{e}-04$ & 16 & $4.5 \mathrm{e}-04$ & $7.519 \mathrm{e}-04$ \\
& 2048 & $3.1 \mathrm{e}-04$ & 15 & $3.8 \mathrm{e}-03$ & $5.7 \mathrm{e}-04$ & 16 & $5.7 \mathrm{e}-04$ & $6.623 \mathrm{e}-04$ \\
& 4096 & $1.1 \mathrm{e}-04$ & 15 & $5.2 \mathrm{e}-03$ & $1.9 \mathrm{e}-04$ & 16 & $1.9 \mathrm{e}-04$ & $6.061 \mathrm{e}-04$ \\
\hline 1 & 1024 & $2.5 \mathrm{e}-05$ & 5 & $1.5 \mathrm{e}-02$ & $1.7 \mathrm{e}-05$ & 9 & $1.7 \mathrm{e}-05$ & $5.495 \mathrm{e}-03$ \\
& 2048 & $1.3 \mathrm{e}-05$ & 5 & $1.7 \mathrm{e}-02$ & $2.3 \mathrm{e}-05$ & 9 & $2.3 \mathrm{e}-05$ & $5.000 \mathrm{e}-03$ \\
& 4096 & $5.7 \mathrm{e}-06$ & 5 & $1.7 \mathrm{e}-02$ & $7.7 \mathrm{e}-06$ & 9 & $7.8 \mathrm{e}-06$ & $4.630 \mathrm{e}-03$ \\
\hline
\end{tabular}

Table 26: Absolute $\ell_{\infty}$ errors for methods listed from left to right: eigenfunction expansion with $m$ terms, Crank-Nicolson, rational Krylov projection with $p$ projection steps, and ode15s with average time step $\Delta t_{\mathrm{avg}}$, for solving (3) to time $t_{f}$ on a $N$-point uniform grid with $u(x, 0)=\sin 2 x, \vec{\alpha}=[1,2,3,4,2,6,4]$ and $\vec{\rho}=[3 / 8,1 / 2,3 / 4,4 / 5,5 / 6,6 / 7]$.

\begin{tabular}{|l|c|c|c|c|c|c|c|c|}
\hline \multicolumn{2}{|c|}{} & \multicolumn{2}{c|}{ eig-exp } & C-N & \multicolumn{2}{c|}{ Krylov } & \multicolumn{2}{c|}{ ode15s } \\
\hline$t_{f}$ & $N$ & time & $m$ & time & time & $p$ & time & $\Delta t_{\text {avg }}$ \\
\hline 0.01 & 1024 & 0.077 & 45 & 0.047 & 0.037 & 28 & 0.047 & $1.724 \mathrm{e}-04$ \\
& 2048 & 0.067 & 45 & 0.322 & 0.322 & 28 & 0.198 & $1.587 \mathrm{e}-04$ \\
& 4096 & 0.099 & 45 & 0.194 & 0.129 & 28 & 0.333 & $1.493 \mathrm{e}-04$ \\
\hline 0.1 & 1024 & 0.017 & 13 & 0.041 & 0.016 & 16 & 0.065 & $1.190 \mathrm{e}-03$ \\
& 2048 & 0.018 & 13 & 0.081 & 0.027 & 16 & 0.091 & $1.124 \mathrm{e}-03$ \\
& 4096 & 0.023 & 13 & 0.159 & 0.051 & 16 & 0.198 & $1.075 \mathrm{e}-03$ \\
\hline 1 & 1024 & 0.008 & 5 & 0.069 & 0.022 & 10 & 0.103 & $9.174 \mathrm{e}-03$ \\
& 2048 & 0.008 & 5 & 0.156 & 0.031 & 10 & 0.187 & $8.772 \mathrm{e}-03$ \\
& 4096 & 0.010 & 5 & 0.159 & 0.035 & 10 & 0.258 & $8.475 \mathrm{e}-03$ \\
\hline
\end{tabular}

Table 27: Execution times (in seconds) for methods listed from left to right: eigenfunction expansion with $m$ terms, Crank-Nicolson, rational Krylov projection with $p$ projection steps, and ode15s with average time step $\Delta t_{\text {avg }}$, for solving (3) to time $t_{f}$ on a $N$-point uniform grid with $u(x, 0)$ from (37), $\vec{\alpha}=[1,2,3,4,2,6,4]$ and $\vec{\rho}=[3 / 8,1 / 2,3 / 4,4 / 5,5 / 6,6 / 7]$.

and their fourier transforms., Journal of Functional Analysis 24(3), 258267.

[18] M. Benedicks, On fourier transforms of functions supported on sets of finite lebesgue measure., J. Math. Anal. Appl. 106(1), 180-183.

[19] J. V. Lambers, Approximate diagonalization of variable-coefficient differential operators through similarity transformations, Computers and Mathematics with Applications 64 (8) (2012) 2575-2593.

[20] M. S. Min, D. Gottlieb, On the convergence of the fourier approximation for eigenvalues and eigenfunctions of discontinuous problems, SIAM J. Numer. Anal. 40 (6) (2003) 2254-2269. 


\begin{tabular}{|l|c|c|c|c|c|c|c|c|}
\hline \multicolumn{2}{|c|}{} & \multicolumn{2}{c|}{ eig-exp } & C-N & \multicolumn{2}{c|}{ Krylov } & \multicolumn{2}{c|}{ ode15s } \\
\hline$t_{f}$ & $N$ & error & $m$ & error & error & $p$ & error & $\Delta t_{\text {avg }}$ \\
\hline 0.01 & 1024 & $1.7 \mathrm{e}-02$ & 45 & $6.6 \mathrm{e}-05$ & $6.5 \mathrm{e}-05$ & 28 & $6.6 \mathrm{e}-05$ & $1.724 \mathrm{e}-04$ \\
& 2048 & $1.7 \mathrm{e}-02$ & 45 & $1.5 \mathrm{e}-04$ & $5.7 \mathrm{e}-05$ & 28 & $5.8 \mathrm{e}-05$ & $1.587 \mathrm{e}-04$ \\
& 4096 & $1.7 \mathrm{e}-02$ & 45 & $1.2 \mathrm{e}-03$ & $2.0 \mathrm{e}-05$ & 28 & $2.0 \mathrm{e}-05$ & $1.493 \mathrm{e}-04$ \\
\hline 0.1 & 1024 & $6.7 \mathrm{e}-04$ & 13 & $2.4 \mathrm{e}-03$ & $5.5 \mathrm{e}-04$ & 16 & $5.5 \mathrm{e}-04$ & $1.190 \mathrm{e}-03$ \\
& 2048 & $4.0 \mathrm{e}-04$ & 13 & $7.5 \mathrm{e}-03$ & $3.5 \mathrm{e}-04$ & 16 & $3.4 \mathrm{e}-04$ & $1.124 \mathrm{e}-03$ \\
& 4096 & $1.4 \mathrm{e}-04$ & 13 & $1.0 \mathrm{e}-02$ & $1.2 \mathrm{e}-04$ & 16 & $1.3 \mathrm{e}-04$ & $1.075 \mathrm{e}-03$ \\
\hline 1 & 1024 & $2.2 \mathrm{e}-05$ & 5 & $3.3 \mathrm{e}-02$ & $2.2 \mathrm{e}-05$ & 10 & $5.2 \mathrm{e}-05$ & $9.174 \mathrm{e}-03$ \\
& 2048 & $1.1 \mathrm{e}-05$ & 5 & $3.7 \mathrm{e}-02$ & $4.2 \mathrm{e}-05$ & 10 & $1.0 \mathrm{e}-04$ & $8.772 \mathrm{e}-03$ \\
& 4096 & $4.9 \mathrm{e}-06$ & 5 & $3.8 \mathrm{e}-02$ & $1.4 \mathrm{e}-05$ & 10 & $3.6 \mathrm{e}-05$ & $8.475 \mathrm{e}-03$ \\
\hline
\end{tabular}

Table 28: Absolute $\ell_{\infty}$ errors for methods listed from left to right: eigenfunction expansion with $m$ terms, Crank-Nicolson, rational Krylov projection with $p$ projection steps, and ode15s with average time step $\Delta t_{\mathrm{avg}}$, for solving (3) to time $t_{f}$ on a $N$-point uniform grid with $u(x, 0)$ from $(37), \vec{\alpha}=[1,2,3,4,2,6,4]$ and $\vec{\rho}=[3 / 8,1 / 2,3 / 4,4 / 5,5 / 6,6 / 7]$.

\begin{tabular}{|l|c|c|c|c|c|c|c|c|}
\hline \multicolumn{2}{|c|}{} & \multicolumn{2}{c|}{ eig-exp } & C-N & \multicolumn{2}{c|}{ Krylov } & \multicolumn{2}{c|}{ ode15s } \\
\hline$t_{f}$ & $N$ & time & $m$ & time & time & $p$ & time & $\Delta t_{\text {avg }}$ \\
\hline 0.01 & 1024 & 0.043 & 27 & 0.106 & 0.047 & 21 & 0.112 & $9.009 \mathrm{e}-05$ \\
& 2048 & 0.044 & 27 & 0.092 & 0.044 & 22 & 0.166 & $7.692 \mathrm{e}-05$ \\
& 4096 & 0.055 & 27 & 0.178 & 0.390 & 22 & 0.414 & $6.897 \mathrm{e}-05$ \\
\hline 0.1 & 1024 & 0.014 & 9 & 0.042 & 0.030 & 13 & 0.146 & $6.410 \mathrm{e}-04$ \\
& 2048 & 0.015 & 9 & 0.087 & 0.028 & 13 & 0.188 & $5.682 \mathrm{e}-04$ \\
& 4096 & 0.018 & 9 & 0.159 & 0.045 & 13 & 0.395 & $5.263 \mathrm{e}-04$ \\
\hline 1 & 1024 & 0.006 & 3 & 0.044 & 0.014 & 10 & 0.113 & $4.808 \mathrm{e}-03$ \\
& 2048 & 0.006 & 3 & 0.106 & 0.016 & 8 & 0.240 & $4.329 \mathrm{e}-03$ \\
& 4096 & 0.006 & 3 & 0.226 & 0.055 & 8 & 0.542 & $4.115 \mathrm{e}-03$ \\
\hline
\end{tabular}

Table 29: Execution times (in seconds) for methods listed from left to right: eigenfunction expansion with $m$ terms, Crank-Nicolson, rational Krylov projection with $p$ projection steps, and ode15s with average time step $\Delta t_{\text {avg }}$, for solving (3) to time $t_{f}$ on a $N$-point uniform grid with $u(x, 0)=\sin 2 x, \vec{\alpha}=[1,5,3,4,2,7,6,8]$ and $\vec{\rho}=$ $[1 / 8,1 / 4,3 / 8,1 / 2,5 / 8,3 / 4,7 / 8]$.

[21] I. Moret, P. Novati, Rd-rational approximation of the matrix exponential operator, BIT 44 (2004) 595-615.

[22] A. Aurko, J. V. Lambers, Computing eigenvalues of 2-d differential operators with piecewise constant coefficients, In preparation.

[23] D. Dereudre, S. Mazzonetto, S. Roelly, Exact simulation of brownian diffusions with drift admitting jumps, SIAM J. Sci. Comput. 39 (3) (2017) A711-A740. 


\begin{tabular}{|l|c|c|c|c|c|c|c|c|}
\hline \multicolumn{2}{|c|}{} & \multicolumn{2}{c|}{ eig-exp } & C-N & \multicolumn{2}{c|}{ Krylov } & \multicolumn{2}{c|}{ ode15s } \\
\hline$t_{f}$ & $N$ & error & $m$ & error & error & $p$ & error & $\Delta t_{\text {avg }}$ \\
\hline 0.01 & 1024 & $3.8 \mathrm{e}-05$ & 27 & $7.9 \mathrm{e}-04$ & $3.4 \mathrm{e}-05$ & 21 & $3.9 \mathrm{e}-05$ & $9.009 \mathrm{e}-05$ \\
& 2048 & $9.2 \mathrm{e}-06$ & 27 & $2.1 \mathrm{e}-03$ & $8.4 \mathrm{e}-06$ & 22 & $1.5 \mathrm{e}-05$ & $7.692 \mathrm{e}-05$ \\
& 4096 & $2.2 \mathrm{e}-06$ & 27 & $3.1 \mathrm{e}-03$ & $2.0 \mathrm{e}-06$ & 22 & $6.4 \mathrm{e}-06$ & $6.897 \mathrm{e}-05$ \\
\hline 0.1 & 1024 & $2.4 \mathrm{e}-06$ & 9 & $9.0 \mathrm{e}-03$ & $8.9 \mathrm{e}-06$ & 13 & $8.6 \mathrm{e}-06$ & $6.410 \mathrm{e}-04$ \\
& 2048 & $6.2 \mathrm{e}-07$ & 9 & $1.1 \mathrm{e}-02$ & $2.2 \mathrm{e}-06$ & 13 & $3.4 \mathrm{e}-06$ & $5.682 \mathrm{e}-04$ \\
& 4096 & $1.8 \mathrm{e}-07$ & 9 & $1.2 \mathrm{e}-02$ & $5.4 \mathrm{e}-07$ & 13 & $1.3 \mathrm{e}-06$ & $5.263 \mathrm{e}-04$ \\
\hline \multirow{2}{*}{0.8} & 1024 & $1.3 \mathrm{e}-09$ & 3 & $3.8 \mathrm{e}-02$ & $5.1 \mathrm{e}-08$ & 10 & $1.7 \mathrm{e}-07$ & $4.808 \mathrm{e}-03$ \\
& 2048 & $4.8 \mathrm{e}-10$ & 3 & $3.9 \mathrm{e}-02$ & $1.3 \mathrm{e}-08$ & 8 & $1.5 \mathrm{e}-07$ & $4.329 \mathrm{e}-03$ \\
& 4096 & $2.7 \mathrm{e}-10$ & 3 & $4.0 \mathrm{e}-02$ & $3.4 \mathrm{e}-09$ & 8 & $1.2 \mathrm{e}-07$ & $4.115 \mathrm{e}-03$ \\
\hline
\end{tabular}

Table 30: Absolute $\ell_{\infty}$ errors for methods listed from left to right: eigenfunction expansion with $m$ terms, Crank-Nicolson, rational Krylov projection with $p$ projection steps, and ode15s with average time step $\Delta t_{\text {avg }}$, for solving (3) to time $t_{f}$ on a $N$-point uniform grid with $u(x, 0)=\sin 2 x, \vec{\alpha}=[1,5,3,4,2,7,6,8]$ and $\vec{\rho}=[1 / 8,1 / 4,3 / 8,1 / 2,5 / 8,3 / 4,7 / 8]$.

\begin{tabular}{|l|c|c|c|c|c|c|c|c|}
\hline \multicolumn{2}{|c|}{} & \multicolumn{2}{c|}{ eig-exp } & C-N & \multicolumn{2}{c|}{ Krylov } & \multicolumn{2}{c|}{ ode15s } \\
\hline$t_{f}$ & $N$ & time & $m$ & time & time & $p$ & time & $\Delta t_{\text {avg }}$ \\
\hline 0.01 & 1024 & 0.042 & 27 & 0.039 & 0.027 & 21 & 0.048 & $1.250 \mathrm{e}-04$ \\
& 2048 & 0.052 & 27 & 0.088 & 0.053 & 21 & 0.142 & $1.176 \mathrm{e}-04$ \\
& 4096 & 0.059 & 27 & 0.360 & 0.128 & 21 & 0.230 & $1.124 \mathrm{e}-04$ \\
\hline 0.1 & 1024 & 0.014 & 9 & 0.052 & 0.018 & 13 & 0.081 & $9.434 \mathrm{e}-04$ \\
& 2048 & 0.015 & 9 & 0.085 & 0.028 & 13 & 0.129 & $9.009 \mathrm{e}-04$ \\
& 4096 & 0.019 & 9 & 0.200 & 0.075 & 13 & 0.586 & $8.696 \mathrm{e}-04$ \\
\hline 1 & 1024 & 0.006 & 3 & 0.050 & 0.013 & 8 & 0.079 & $7.752 \mathrm{e}-03$ \\
& 2048 & 0.006 & 3 & 0.183 & 0.063 & 8 & 0.250 & $7.463 \mathrm{e}-03$ \\
& 4096 & 0.007 & 3 & 0.211 & 0.043 & 8 & 0.646 & $7.246 \mathrm{e}-03$ \\
\hline
\end{tabular}

Table 31: Execution times (in seconds) for methods listed from left to right: eigenfunction expansion with $m$ terms, Crank-Nicolson, rational Krylov projection with $p$ projection steps, and ode15s with average time step $\Delta t_{\text {avg }}$, for solving (3) to time $t_{f}$ on a $N$-point uniform grid with $u(x, 0)$ from $(37), \vec{\alpha}=[1,5,3,4,2,7,6,8]$ and $\vec{\rho}=$ $[1 / 8,1 / 4,3 / 8,1 / 2,5 / 8,3 / 4,7 / 8]$.

\begin{tabular}{|l|c|c|c|c|c|c|c|c|}
\hline \multicolumn{2}{|c|}{} & \multicolumn{2}{c|}{ eig-exp } & C-N & \multicolumn{2}{c|}{ Krylov } & \multicolumn{2}{c|}{ ode15s } \\
\hline$t_{f}$ & $N$ & error & $m$ & error & error & $p$ & error & $\Delta t_{\text {avg }}$ \\
\hline 0.01 & 1024 & $1.9 \mathrm{e}-05$ & 27 & $3.5 \mathrm{e}-03$ & $1.3 \mathrm{e}-05$ & 21 & $4.0 \mathrm{e}-05$ & $1.250 \mathrm{e}-04$ \\
& 2048 & $4.6 \mathrm{e}-06$ & 27 & $1.1 \mathrm{e}-02$ & $3.2 \mathrm{e}-06$ & 21 & $2.9 \mathrm{e}-05$ & $1.176 \mathrm{e}-04$ \\
& 4096 & $1.3 \mathrm{e}-06$ & 27 & $1.6 \mathrm{e}-02$ & $8.9 \mathrm{e}-07$ & 21 & $2.5 \mathrm{e}-05$ & $1.124 \mathrm{e}-04$ \\
\hline 0.1 & 1024 & $3.0 \mathrm{e}-06$ & 9 & $4.9 \mathrm{e}-02$ & $1.5 \mathrm{e}-06$ & 13 & $7.0 \mathrm{e}-05$ & $9.434 \mathrm{e}-04$ \\
& 2048 & $7.6 \mathrm{e}-07$ & 9 & $5.7 \mathrm{e}-02$ & $3.8 \mathrm{e}-07$ & 13 & $5.9 \mathrm{e}-05$ & $9.009 \mathrm{e}-04$ \\
& 4096 & $1.9 \mathrm{e}-07$ & 9 & $5.9 \mathrm{e}-02$ & $9.1 \mathrm{e}-08$ & 13 & $5.1 \mathrm{e}-05$ & $8.696 \mathrm{e}-04$ \\
\hline 1 & 1024 & $1.1 \mathrm{e}-08$ & 3 & $1.4 \mathrm{e}-01$ & $6.6 \mathrm{e}-08$ & 8 & $1.6 \mathrm{e}-05$ & $7.752 \mathrm{e}-03$ \\
& 2048 & $2.9 \mathrm{e}-09$ & 3 & $1.4 \mathrm{e}-01$ & $1.6 \mathrm{e}-08$ & 8 & $1.6 \mathrm{e}-05$ & $7.463 \mathrm{e}-03$ \\
& 4096 & $9.0 \mathrm{e}-10$ & 3 & $1.4 \mathrm{e}-01$ & $4.0 \mathrm{e}-09$ & 8 & $1.8 \mathrm{e}-05$ & $7.246 \mathrm{e}-03$ \\
\hline
\end{tabular}

Table 32: Absolute $\ell_{\infty}$ errors for methods listed from left to right: eigenfunction expansion with $m$ terms, Crank-Nicolson, rational Krylov projection with $p$ projection steps, and ode15s with average time step $\Delta t_{\text {avg }}$, for solving (3) to time $t_{f}$ on a $N$-point uniform grid with $u(x, 0)$ from $(37), \vec{\alpha}=[1,5,3,4,2,7,6,8]$ and $\vec{\rho}=[1 / 8,1 / 4,3 / 8,1 / 2,5 / 8,3 / 4,7 / 8]$. 

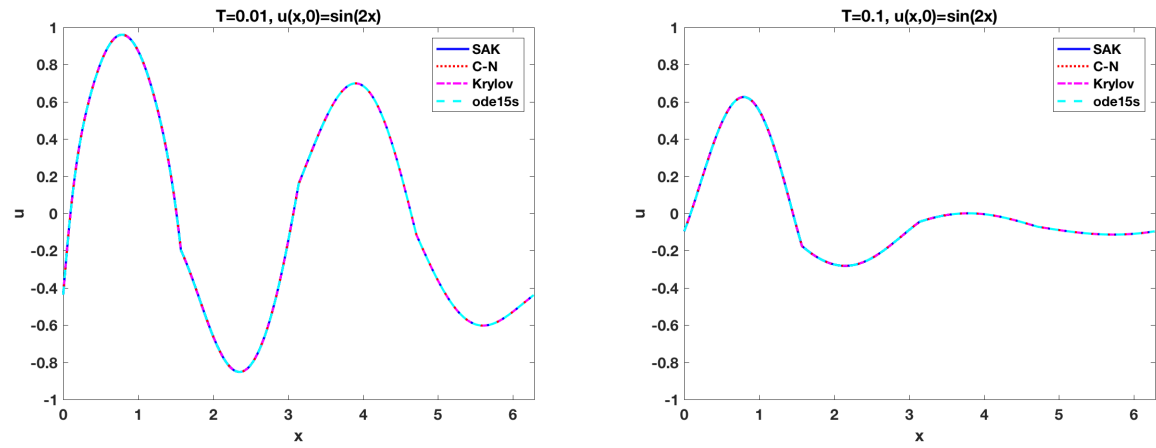

Figure 11: Solutions of $(3), u(x, 0)=\sin 2 x$, computed at $t_{f}=0.01,0.1$ on a uniform grid with $N=1,024$ points. The coefficient $\alpha^{2}(x)$ is defined by (1) with $\vec{\alpha}=[1,2,3,4]$, $\vec{\rho}=[1 / 4,1 / 2,3 / 4]$.
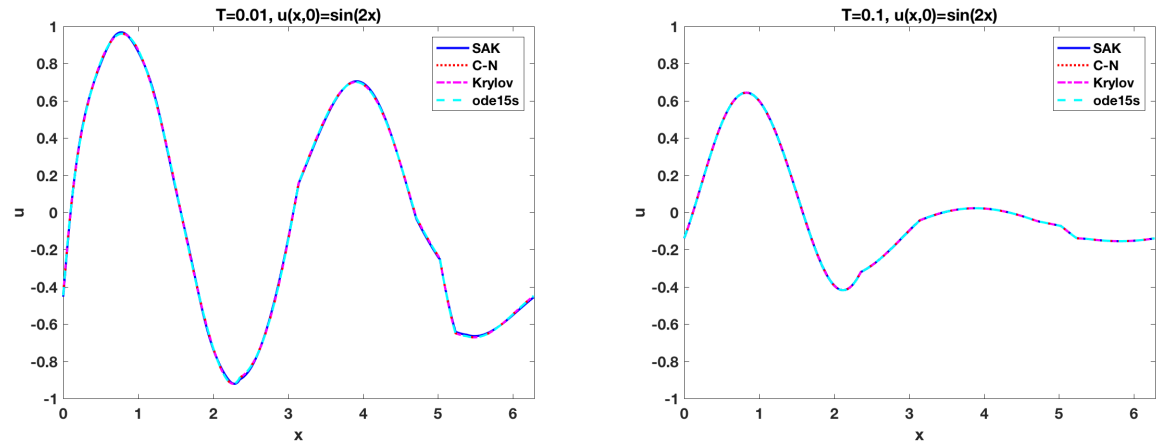

Figure 12: Solutions of $(3), u(x, 0)=\sin 2 x$, computed at $t_{f}=0.01,0.1$ on a uniform grid with $N=1,024$ points. The coefficient $\alpha^{2}(x)$ is defined by (1) with $\vec{\alpha}=[1,2,3,4,2,6,4]$, $\vec{\rho}=[3 / 8,1 / 2,3 / 4,4 / 5,5 / 6,6 / 7]$.
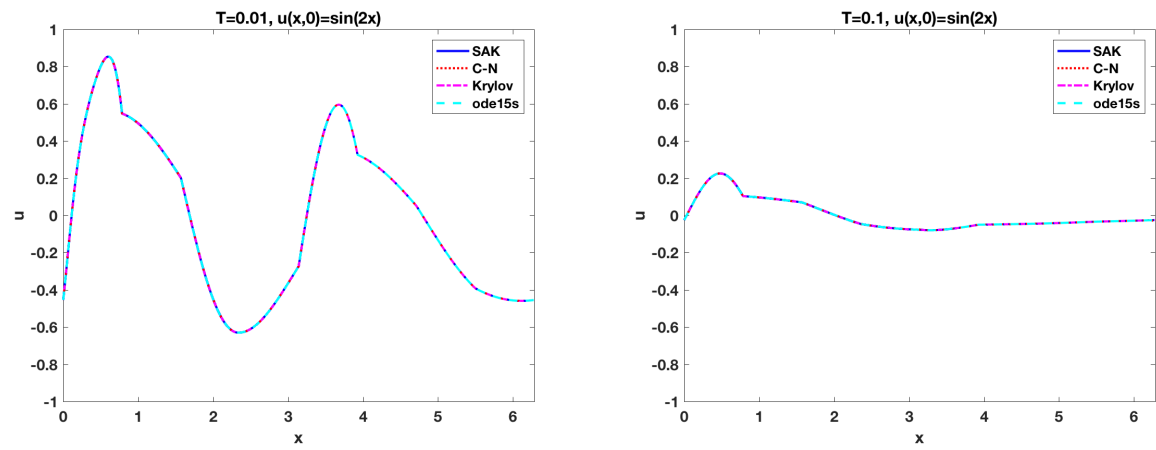

Figure 13: Solutions of $(3), u(x, 0)=\sin 2 x$, computed at $t_{f}=0.01,0.1$ on a uniform grid with $N=1,024$ points. The coefficient $\alpha^{2}(x)$ is defined by (1) with $\vec{\alpha}=[1,5,3,4,2,7,6,8]$, $\vec{\rho}=[1 / 8,1 / 4,3 / 8,1 / 2,5 / 8,3 / 4,7 / 8]$. 

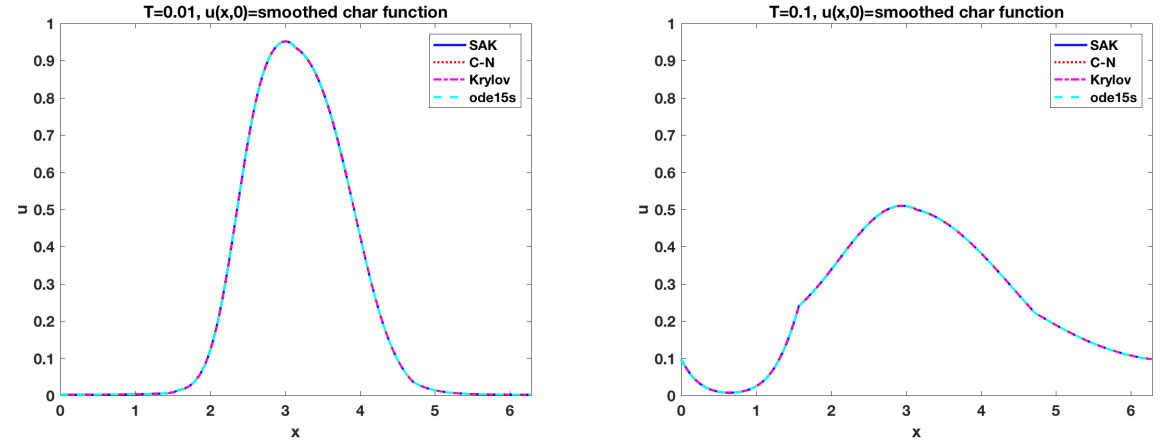

Figure 14: Solutions of $(3), u(x, 0)$ from (37), computed at $t_{f}=0.01,0.1$ on a uniform grid with $N=1,024$ points. The coefficient $\alpha^{2}(x)$ is defined by (1) with $\vec{\alpha}=[1,2,3,4]$, $\vec{\rho}=[1 / 4,1 / 2,3 / 4]$.
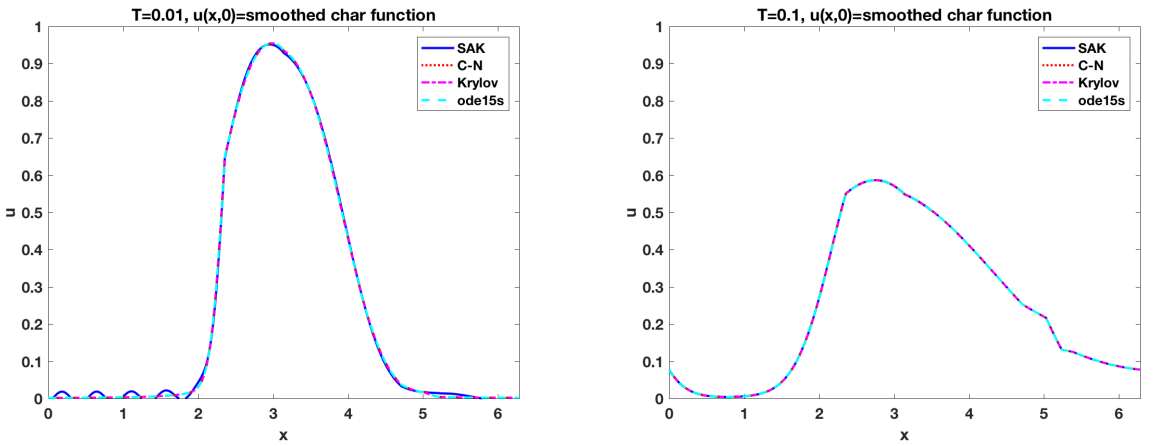

Figure 15: Solutions of $(3), u(x, 0)$ from $(37)$, computed at $t_{f}=0.01,0.1$ on a uniform grid with $N=1,024$ points. The coefficient $\alpha^{2}(x)$ is defined by (1) with $\vec{\alpha}=[1,2,3,4,2,6,4]$, $\vec{\rho}=[3 / 8,1 / 2,3 / 4,4 / 5,5 / 6,6 / 7]$.
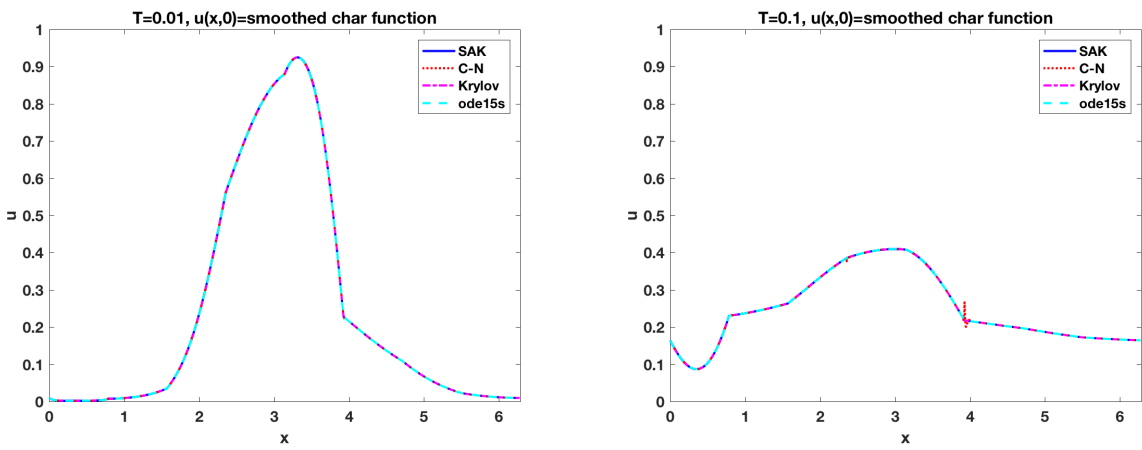

Figure 16: Solutions of $(3), u(x, 0)$ from $(37)$, computed at $t_{f}=0.01,0.1$ on a uniform grid with $N=1,024$ points. The coefficient $\alpha^{2}(x)$ is defined by (1) with $\vec{\alpha}=[1,5,3,4,2,7,6,8]$, $\vec{\rho}=[1 / 8,1 / 4,3 / 8,1 / 2,5 / 8,3 / 4,7 / 8]$. 